\title{
A THIRD UPDATE OF THE STATUS OF THE 3CR SOURCES: FURTHER NEW REDSHIFTS AND NEW IDENTIFICATIONS OF DISTANT GALAXIES*
}

\author{
HYRON SPINRAD, $†$ S. DJORGOVSKI, $\ddagger$ J. MARR, AND L. AGUILAR \\ Department of Astronomy, University of California, Berkeley, California 94720 \\ Received 1985 May 21
}

\begin{abstract}
We present the third major update of new optical and radio data for the strong sources in the Revised Third Cambridge Catalogue (hereafter 3CR). New positions, redshifts, magnitudes, and identifications have been included as well as some radio data for the sample of 298 extragalactic 3CR sources. A new and extended bibliography of optical and radio data is included.

The 3CR sources have now reached an almost satisfactory state of optical identification; $91 \%$ of the sources at $|b| \geqslant 10^{\circ}$ now have firm identifications and we have spectra for most of them. The few remaining faint objects at high latitudes are, of course, important, as they tend to be the most luminous radio sources known. Full completion of the 3CR data will aid in reducing the residual uncertainties at the luminous end of the evolving radio luminosity function.

Finally, we present some new identifications and new spectroscopic data for 29 faint 3CR galaxies, and one quasar.

Key words: radio galaxies-3CR catalog_-galactic redshifts
\end{abstract}

\section{Introduction}

The previous two 3CR status reviews (Smith, Spinrad, and Smith 1976; Smith and Spinrad 1980) reviewed and tabulated the optical information on the extragalactic 3CR sources, using the optical data assembled with the technology of the 1970s. The recent advent of VLA radio maps and central component positions, and new optical CCD detectors for both imaging and spectroscopy, has rapidly improved our ability to detect, indentify, and obtain redshifts for very faint $3 \mathrm{CR}$ candidates. Thus the catalog, even at faint optical levels, is nearing a satisfactory level of completeness.

In this paper we include the old radio positions for most of the unidentified sources from Bennett (1962). Some of these radio positions are quite poor (especially those at low galactic latitudes) and considerable future improvement is needed to bring them up to modern quality; the VLA can do the job.

We added one new source, 3C292, strong enough for 3CR inclusion (cf. Laing, Riley, and Longair 1983, hereafter LRL83). We have retained a few previously included 3CR sources, which turned out, after critical examination of their radio properties, to be resolved doubles or sources weaker than the (old) nominal cutoff of a $9 \mathrm{Jy}$ at $178 \mathrm{MHz}$. This decision was mainly one of historical continuity, so our sample is "softer" than that of LRL83.

* Based in part on research done at Lick Observatory.

†Visiting Observer, Kitt Peak National Observatory, National Optical Astronomy Observatories, operated by AURA, Inc., under contract with the NSF.

$\ddagger$ Visiting Student, Kitt Peak National Observatory, National Optical Astronomy Observatories, operated by AURA, Inc., under contract with the NSF.

\section{The Identification Content of the Present Listing}

The 3CR catalog of 298 extragalactic sources contains the following proportions of safely identified objects:

195 radio galaxies (including the $\mathrm{N}$-Galaxies) $(=65.4 \%)$

53 radio quasars (including the BL-Lac object $3 \mathrm{C} 66$ )

$$
\text { (=17.8\%) }
$$

There are twelve identifications which we consider as yet unconfirmed (they are probably also mostly galaxies), and there are 38 sources which are still unidentified. Of these last 38,24 sources lie at $|b|<10^{\circ}$, and will be difficult objects until the advent of routine $2 \mu-5 \mu$ IR imaging. We also note that many of the obviously obscured sources also need better, modern radio maps, presumably obtainable with the VLA. Some may have central components, centered precisely on the optical candidates (cf. Laing, Owen, and Puschell 1983, hereafter LOP). The future IR observers can take advantage of the improved positions. The 14 unidentified 3CR sources at higher latitudes should also be systematically remapped with a large dynamic-range radio synthesis system. After that effort, reimaging in the optical with a CCD camera at a good seeing site could push the optical detection limit close to $V=25$, if necessary. It will be important to check whether these few unidentified, presumably very faint sources, could be distant galaxies with $z \geqslant 2.0$. If so, they might be very red ("old and dead", as far as star formation is concerned) galaxies, following the most passive evolutionary models of Bruzual (1983), as suggested for 3C437 and 3C470 by Lilly (1983) and Lilly and Longair (1985). If that scenario is correct, their detection will actually be easier at $2 \mu$ or $3 \mu$ in the near-IR, than in the visual-red regime.

The present attention of faint galaxy observers in the remaining powerful 3CR sources has lead to numerous 
new identifications, and a good redshift success rate, since most of the strong radio galaxies show moderate/ strong emission lines (Spinrad and Djorgovski 1984a, $b$; Perryman et al. 1984). The 23 unconfirmed and/or unidentified high-latitude sources comprise only $9 \%$ of the total extragalactic sample at $|b| \geqslant 10^{\circ} ; 91 \%$ have now been well identified and most have optical spectra, although a few faint objects have defied our attempted redshift measurements (e.g., $3 \mathrm{C} 322, \mathrm{~V} \approx 23.5$; no obvious emission lines).

In the 3CR listing (section VI of this paper), we note that the 20 most-distant sources tabulated $(1.2 \leqslant z \leqslant 2.01)$ are a mixture of quasars and radio galaxies, with $\langle z\rangle_{\mathrm{med}}=$ 1.5 and $S_{178} \approx 13 \mathrm{Jy}$. They are extremely powerful radio sources with $P_{178}>10^{27} \mathrm{WHz}^{-1} \mathrm{sr}^{-1}$, among the most luminous sources in the radio region. They share the general cosmic evolution of QSOs, with a $\left(V / V_{\text {max }}\right)$ ratio $\simeq$ 0.67 (LRL83). 3C256 (Spinrad and Djorgovski 1984b) still has the highest galaxy redshift, $z=1.819$, although several fainter galaxies (3C65, 3C241) have been observed and had smaller redshifts. Windhorst (1984) has speculated that there might be a redshift limit of about 2 on radio galaxies of high flux density (3CR class).

Looking at the distribution sorted by radio flux density at $178 \mathrm{MHz}\left(S_{178}\right)$, we find that the brightest 20 identified sources (working down from Cygnus A) have $S_{178} \sim 100 \mathrm{Jy}$ and a median redshift, $\langle z\rangle_{\text {med }} \simeq 0.07$, with a large dispersion. The weakest 20 identified sources, with $S_{178} \simeq 8 \mathrm{Jy}$ show $\langle z\rangle_{\text {med }} \simeq 0.32$, significantly larger than 0.07 ; this is the sense of the difference anticipated for a set of objects with a small dispersion in $M_{v}$ (optically standard candles) for the $\mathrm{gE}$ galaxies which represent a majority of the 3CR identifications (Sandage 1972). Figure 1 shows the present redshift distributions for the 3CR sample. The median redshift for all the radio galaxies (excluding the $\mathrm{N}$

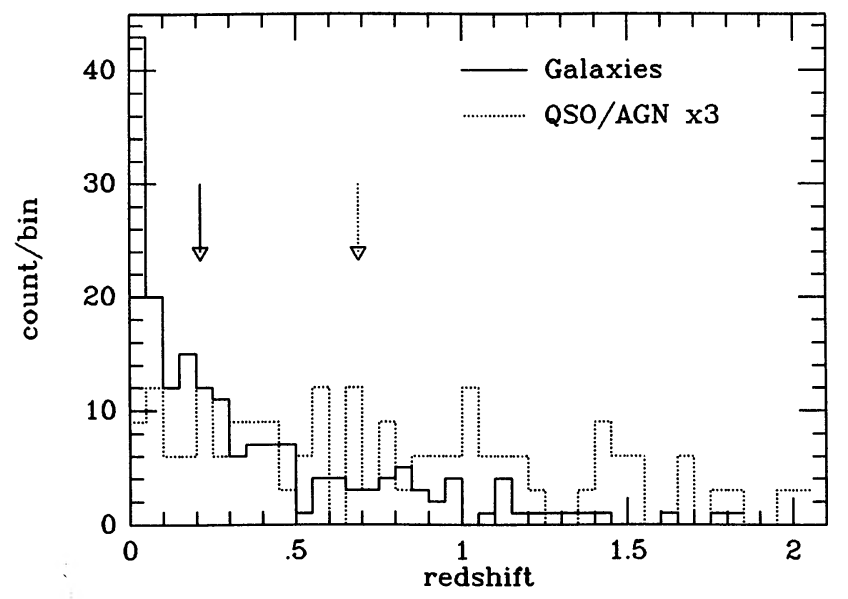

FIG. 1-Redshift distributions for the 3CR sources observed spectroscopically. The solid line corresponds to the radio-galaxies (excluding the $\mathrm{N}$-galaxies), and the dotted line to the QSOs, $\mathrm{N}$-galaxies, and BL Lac objects. The bin counts for the latter group were multiplied by 3 for clarity. The arrows mark the sample medians. galaxies) is 0.2145 , and for the mix of QSOs, $\mathrm{N}$ galaxies, and BL Lacertae objects, it is 0.691 .

We confirm another statistical distinction, long known in general, that the source spectral index, (column (7) in the listing) is steeper $(\alpha=0.95)$ for the most luminous radio galaxies $(\langle z\rangle \simeq 1.4)$ than it is for the weaker $3 \mathrm{CR}$ galaxies at $\langle z\rangle \simeq 0.03(\alpha=0.72)$. Figure 2 shows the radio spectral index as a function of redshift for the sample of 3CR radio-galaxies (excluding the $\mathrm{N}$-galaxies). A slight increasing trend may be caused by the radio-equivalent of the K-correction-the radio spectra tend to be curved. Another possibility is that there is some evolution of the properties of radio-sources with redshift.

Completion of the whole 3CR data set is an appealing and approaching goal; complete information on the brightest radio sources will solidly constrain the cosmic epoch of the evolution of the most powerful end of the extragalactic radio source luminosity function (cf. Peacock 1985; Wall and Peacock 1984; Condon 1984).

\section{Some Individual Radio Galaxies of Note}

We present here direct images and identifications for two faint and distant radio galaxies. They are 3C297 (Fig. 3) (initially identified by Browne, private communication; which we confirm), and 3C 305.1 (Fig. 4), identified by H. E. Smith and E. M. Burbidge. Neither galaxy received much attention before 1984. These two radio galaxies do not have modern radio maps, but we feel their identifications to be secure, partly because they are spectroscopically extreme cases of AGN.

The 3C297 image is a gray-scale representation of a portion of a Kitt Peak CCD frame, obtained in mediocre seeing on 1984 April 2 UT by two of us (H.S., S.D.). The detector was an $800 \times 800 \mathrm{TI}$ chip, at the prime focus of the Mayall 4-m telescope.

3C297 may be designated as an N-type galaxy, as its

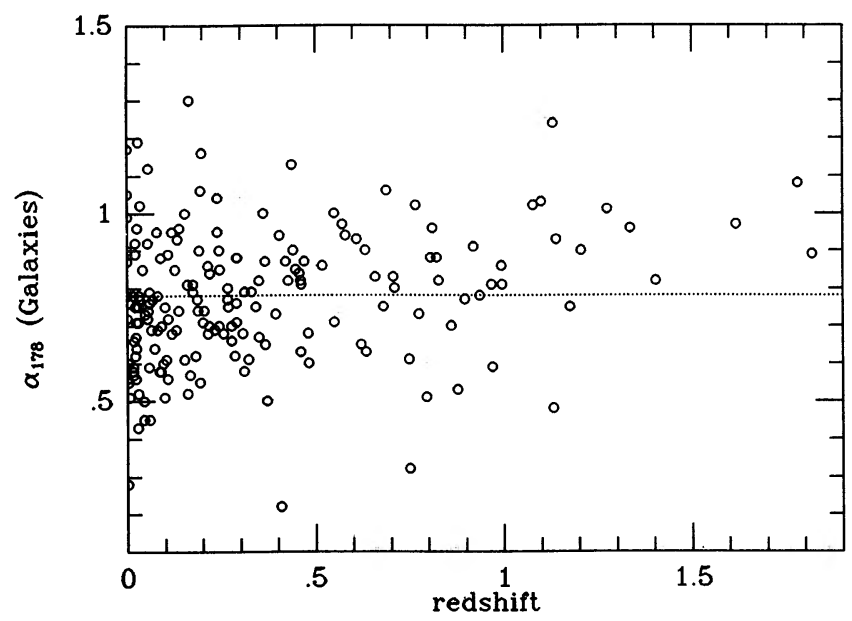

FIG. 2-Radio spectral index as a function of redshift for the 3CR radio-galaxies (excluding the $\mathrm{N}$-galaxies). The dotted line represents the sample median. 
nucleus is well defined, despite the degraded seeing during the exposure-its radial profile shows a definite core-halo structure.

Spectroscopy of 3C297, obtained at Kitt Peak with the

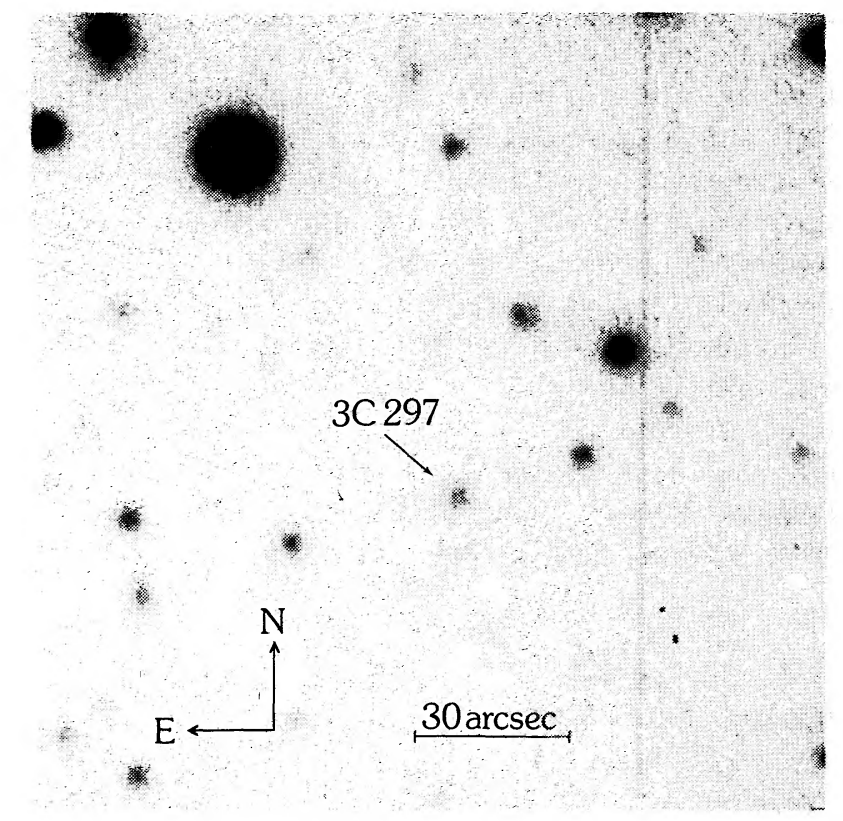

FIG. 3-A grey-scale representation of a portion of a Kitt Peak 4-m CCD frame of 3C297 ( $R$-band), obtained in mediocre seeing on 1984 April 2 UT. 3C 297 is the faint N-galaxy image marked with an arrow.
Cryogenic Camera yielded an emission-line redshift of $z$ $=1.406$; this is, of course, one of the largest measured for a radio galaxy. This $\mathrm{N}$-galaxy shows an emission spectrum with moderately strong high-ionization emission lines.

3C 305.1 was photographed by Dr. Smith at the Kitt Peak 4-m reflector prime focus with a baked (red-sensitive) IIIa-F/GG-495 emulsion-filter combination. A portion of his plate is reproduced here (Fig. 4); the radio galaxy image appears asymmetric, and a deeper CCD frame would be desirable to check on possible appendages to this distant radio galaxy.

The 3C305.1 spectrum was observed in April and May 1984; we used the new CCD spectrograph developed by J. S. Miller and L. Robinson on the Shane 3-m reflector for the Lick spectra, while the higher $S / N$ ratio Kitt Peak spectrogram (see Fig. 3) was obtained with the Cryogenic Camera on the Mayall 4-m reflector. The visual-red spectrum of 3C 305.1 (Fig. 5) shows an enormously strong [OII] $\lambda 3727$, strong CII] $\lambda 2326$, and moderate $\mathrm{Mg}$ II $\lambda 2799$ emission. These lines are typical for low-ionization, powerful radio galaxies (Spinrad and Djorgovski 1985). The redshift measured for 3C305.1 is $z=1.132$.

\section{26 Additional Galaxy/QSO Redshifts}

Over the last few years, Spinrad and Djorgovski have obtained redshifts for many 3CR candidates, and some of them have not yet been published. We list in Table I 29 new and/or confirming redshifts for 3CR galaxies, and one

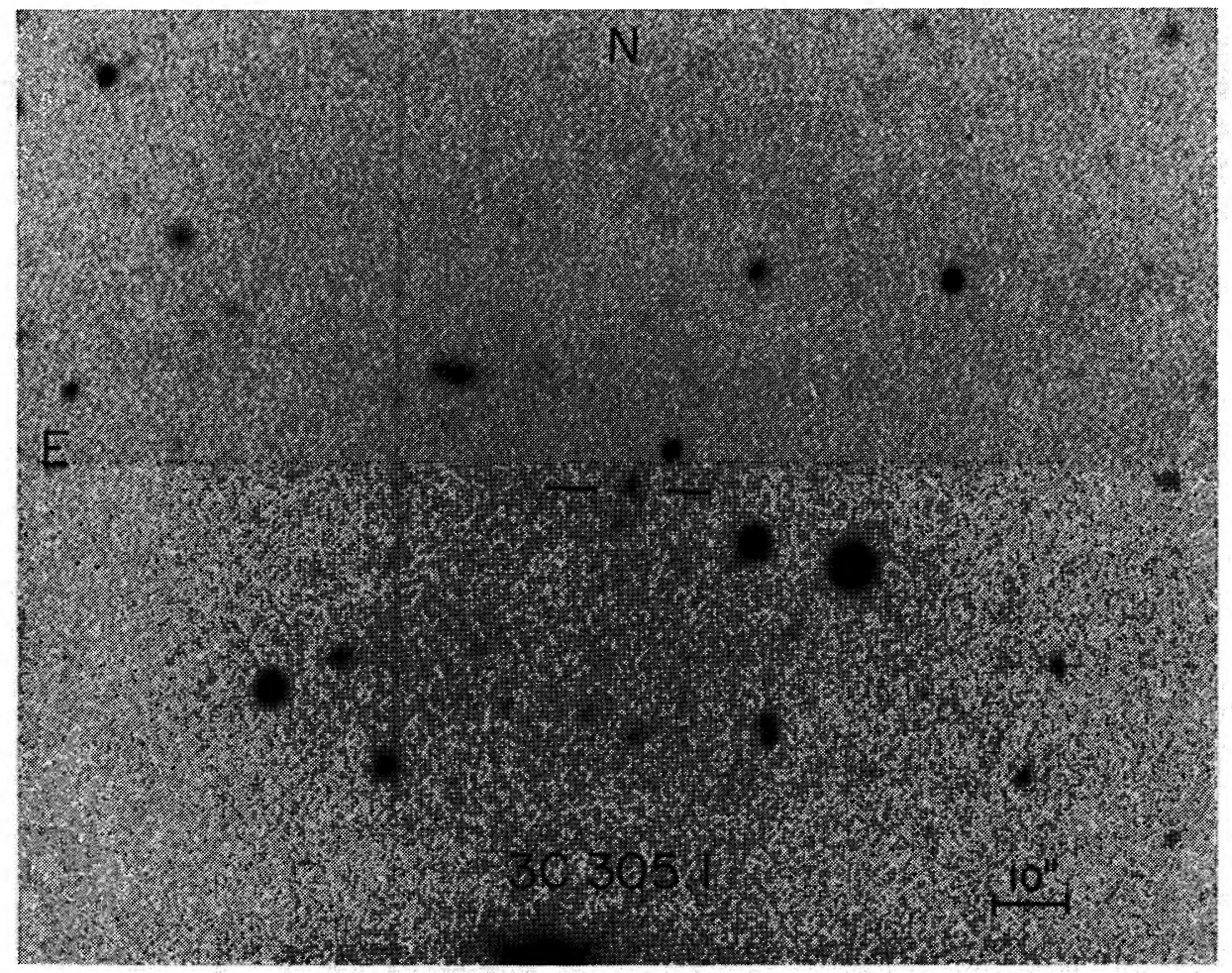

FIG. 4-A portion of a red (IIIa-F) plate showing 3C305. 1, a radio galaxy at $z=1$.132. The original plate was obtained at the 4-m Mayall reflector by Dr. H. E. Smith. 


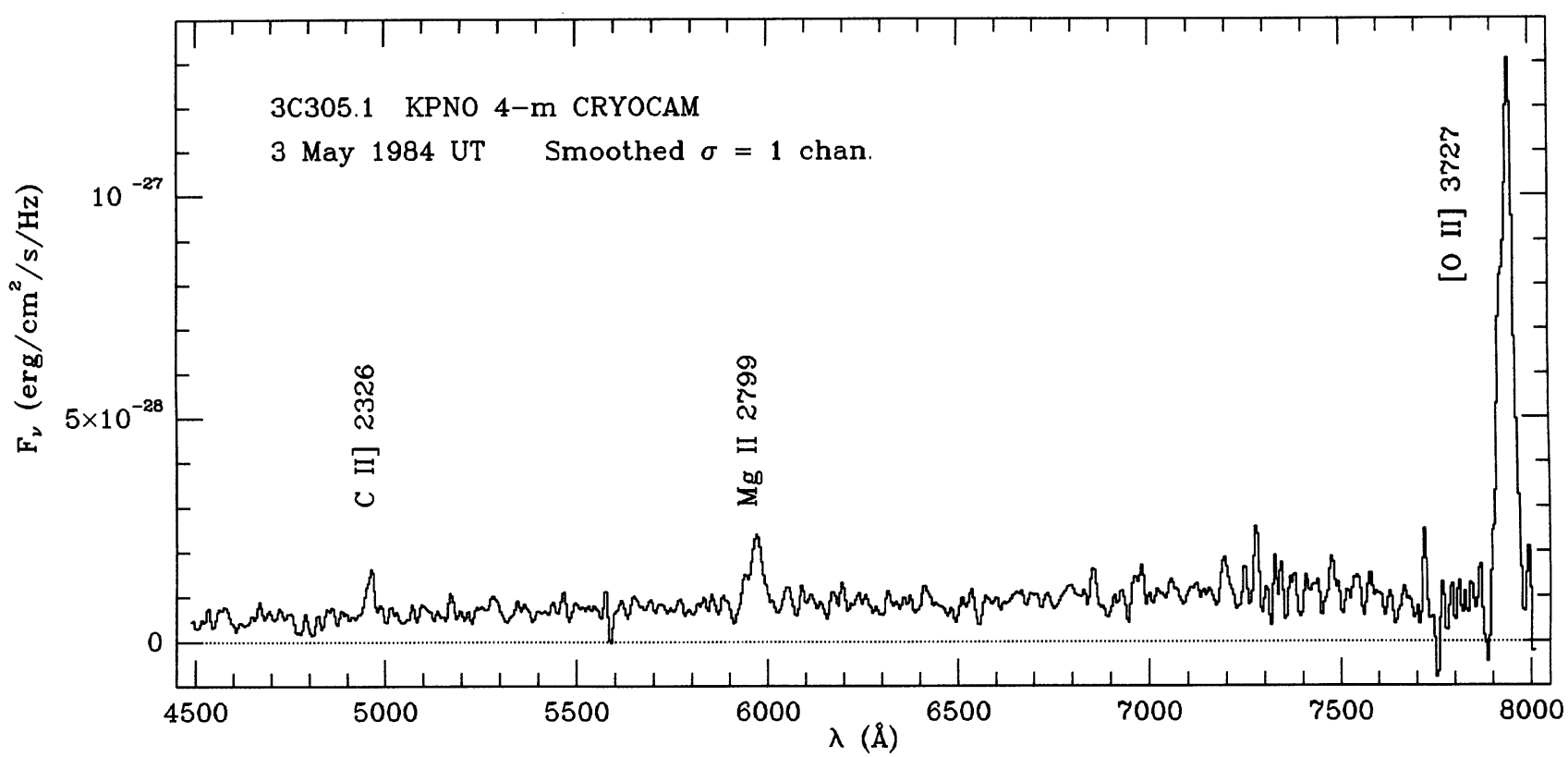

FIG. 5-The low-resolution spectrum of 3C305.1, obtained by Spinrad and Djorgovski at Kitt Peak with the Cryogenic camera. Note the enormous $[\mathrm{OII}] \lambda_{0} 3727$ emission and the moderate (low-ionization) emission lines due to $\mathrm{Mg}$ II $\lambda 2799$ and $\left.\mathrm{CII}\right] \lambda 2326$, at $z_{\mathrm{e}}=1.132$ for this faint radio galaxy.

TABLE I

New Redshifts for 30 3CR objects

\begin{tabular}{|c|c|c|}
\hline 3CR非 & Morph. Type & $\mathrm{z}$ \\
\hline $\begin{array}{l}34 \\
43\end{array}$ & $\begin{array}{l}\text { Gal. } \\
\text { OSO: }\end{array}$ & $\begin{array}{l}0.6897 \\
1.47\end{array}$ \\
\hline 44 & Ga1. & 0.66 \\
\hline 49 & Gal. & 0.6207 \\
\hline 52 & Gal. & 0.285 \\
\hline 54 & Gal. & 0.8274 \\
\hline 61.1 & Gal. & 0.186 \\
\hline 65 & Gal. & 1.176 \\
\hline 103 & Gal. & 0.33 \\
\hline 165 & Gal. & 0.29 \\
\hline 172 & Gal. & 0.5191 \\
\hline 175.1 & Gal. & 0.920 \\
\hline 180 & Gal. & 0.22 \\
\hline 208.1 & $\mathrm{~N}-\mathrm{Gal} ?$ & 1.02 \\
\hline 220.1 & Gal. & 0.61 \\
\hline 220.3 & Gal. & 0.685 \\
\hline $225 B$ & Gal. & 0.58 \\
\hline 226 & Gal. & 0.82 \\
\hline 239 & Gal. & 1.781 \\
\hline 247 & Gal. & 0.7489 \\
\hline 263.1 & Gal. & 0.366 \\
\hline 268.1 & Ga1? & 0.97 \\
\hline 277.2 & Gal. & 0.766 \\
\hline 280 & Gal. & 0.996 \\
\hline 292 & Gal. & 0.71 \\
\hline 297 & $\mathrm{~N}-\mathrm{Gal}$. & 1.4061 \\
\hline 305.1 & Gal. & 1.132 \\
\hline 325 & $\mathrm{~N}-\mathrm{Gal}$. & 0.86 \\
\hline 327.1 & Gal. & 0.4628 \\
\hline 337 & Gal. & 0.635 \\
\hline
\end{tabular}

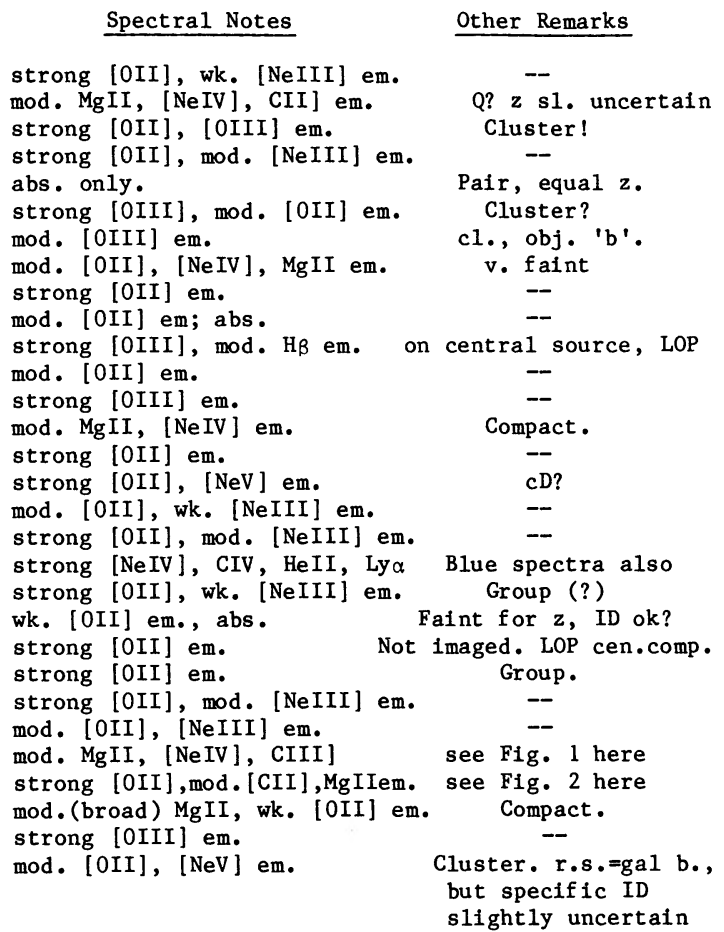

quasar. Spectroscopic measures like these make for real progress in physical understanding of the radio sources; they are an important part of this paper.

\section{Sources with Positional Disagreements or Particular Problems}

Several of our unidentified or uncertain 3CR sources 
have unusual problems; 3C294 is apparently obscured by a galactic star right on the LRL83 position; the optical candidate for $3 \mathrm{C} 225 \mathrm{~A}$ is about $3^{\prime \prime}$ from the radio sources and this (19.5?) faint galaxy may not be the correct identification; (Riley private communication); 3C 250 is some 3" off the wide lobes radio axis and is thus a less secure identification than desired (Gunn et al. 1981), and 3C 194 has a substantial positional offset from the radio position, despite its reasonable optical luminosity (for a radio galaxy at $z=0.31$ ).

Also, since the last edition, a few redshifts have been revised; the most important recent change is the "demise" of 3C 427. 1 (Spinrad 1985) to $z=0.572$. In a catalog of this size some minor problems are inevitable, and its unlikely that all the discrepancies will be easily removed in the next years. These problem sources are, fortunately, only a minor headache to us now.

\section{The 3CR Listings}

Our computer listing is broken into five sections, as before (Smith and Spinrad 1980), with two reference listings. The senior author intends to continue his update of the 3CR data, so contributed future new data on these sources will be gratefully incorporated and privately circulated, as circumstances dictate.

We wish to acknowledge considerable help from many interested astronomers; in particular we thank Drs. S. Lilly, J. Riley, R. Laing, F. Owen, Wil van Breugel, S. Wyckoff, M. Wagner, J. Stocke, I. Browne, and especially H. E. Smith for advice and unpublished data of all sorts. We also thank Doug Baker and Mike Strauss for help with the data reduction.

H. Spinrad's research was supported by a grant from NSF(AST 81-16125). S. Djorgovski was supported by UCB Graduate Fellowship. L. Aguilar was supported by the NSF grant above, as was J. Marr.

\section{REFERENCES}

Bennett, A. S. 1962, Mem. R.A.S. 68, 163.

Bruzual, G. 1983, Ap. J. 273, 105.

Condon, J. J. 1984, Ap. J. 284, 44.

Gunn, J. E., Hoessel, J. G., Westphal, J. A., Perryman, M. A. C., and Longair, M. S. 1981, M.N.R.A.S. 194, 111.

Laing, R. A., Owen, E., and Puschell, J. 1983 (unpublished preprint). (LOP)

Laing, R. A., Riley, J. M., and Longair, M. S. 1983, M.N.R.A.S. 204, 151. (LRL83)

Lilly, S. 1983, Ph. D. Thesis, University of Edinburgh.

Lilly, S., and Longair, M. S. 1984, M.N.R.A.S. 211, 833.

Peacock, J. 1985, M.N.R.A.S. (in press).

Perryman, M. A. C. Lilly, S. J., Longair, M. S., and Downes, A. J. B. 1984, M.N.R.A.S. 209, 159.

Sandage, A. R. 1974, Ap. J. 178, 25.

Smith, H. E., and Spinrad, H. 1980, Pub. A.S.P. 92, 553.

Smith, H. E., Spinrad, H., and Smith, E. O. 1976, Pub. A.S.P. 88, 621. Spinrad, H: 1985, Ap. J. (in press).

Spinrad, H., and Djorgovski, S. 1984a, Ap. J. (Letters) 280, L9.

Spinrad, H., and Djorgovski, S. 1984b, Ap. J. (Letters) 285, L49.

Spinrad, H., and Djorgovski, S. 1985, Ap. J. (in preparation).

Wall, J., and Peacock, J. 1985, M.N.R.A.S. 216, 173.

Windhorst, R. 1984, Ph.D. Thesis, Leiden University.

Note added in press: As this issue goes to print, we note that 3C410 may now be unequivocably identified with a galaxy; its redshift is $z=0.248$. 


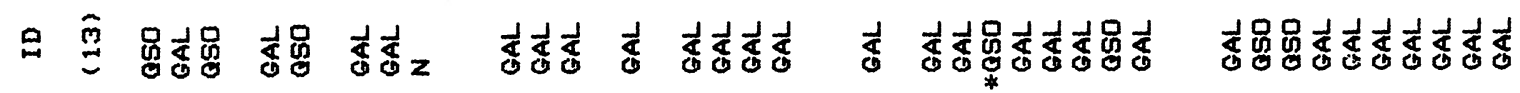

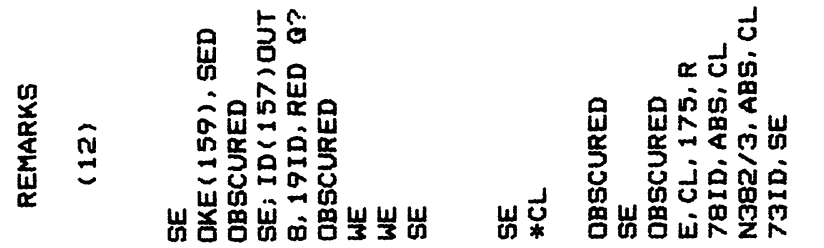

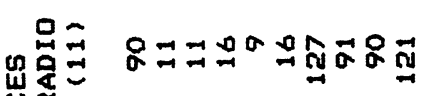

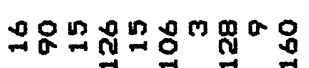

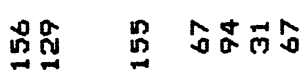

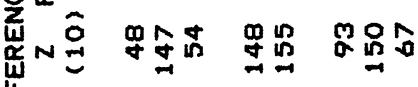

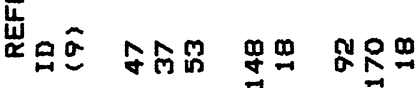

过宁舟

志新

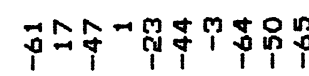

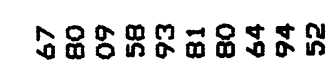

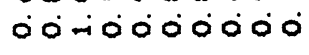

ú $\quad$ úd

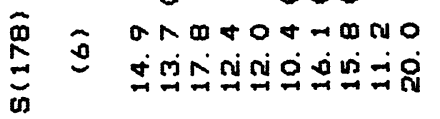

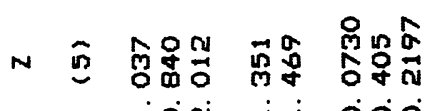
Tion it o0

柕

量
ํํㅇำ

000 00.000

ú $\quad$ ú

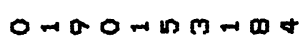

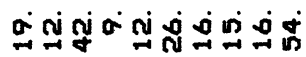

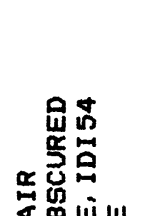

热

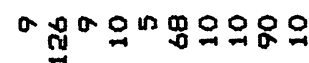

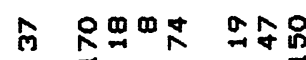

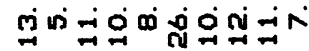
000 0 0000

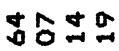
苨 तี क मे 잉

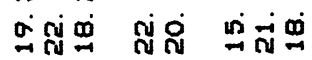

is

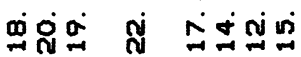

a 0 लู

Ha- m a d a d

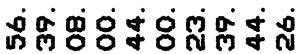

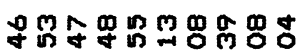

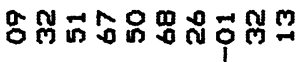

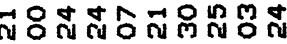

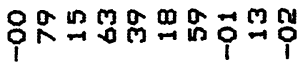
ot un no nom

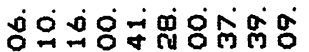
คन

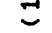

趌嘿

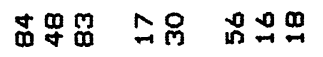

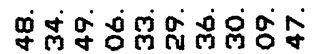

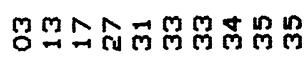
$8: 8: 8: 8: 8:$

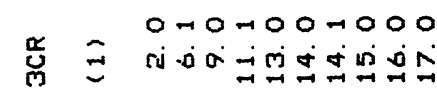

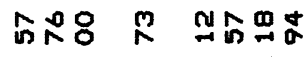

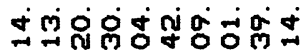

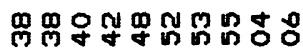
88888888 혀

$000-000000$

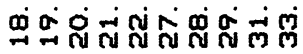

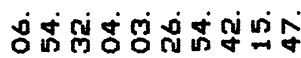

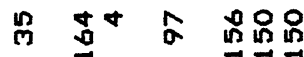

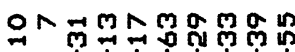

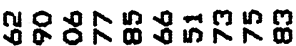

OO-T000000 ఏ

ond in

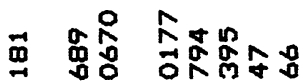
- 0ं iótió

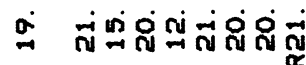

(1) in $\operatorname{con}$ an

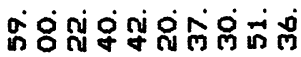

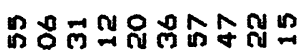

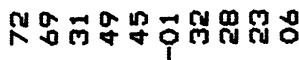

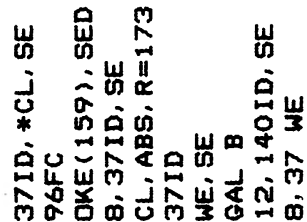

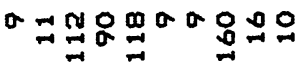

品品的品品品品学品品

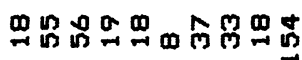

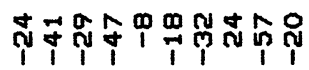

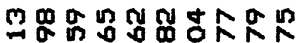
- 000000 . $00^{\circ} 0^{\circ}$ แัứ ú N t O m n m m n N N

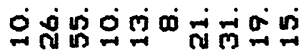

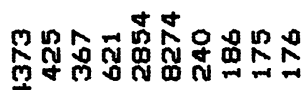
.00000000.

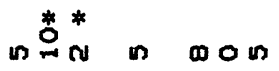

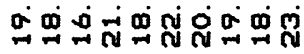

0000 mon

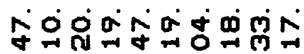

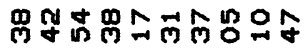

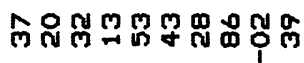

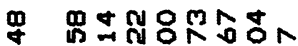

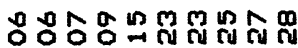

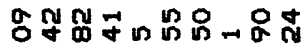

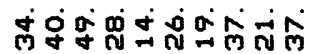

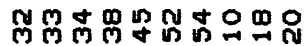

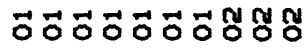

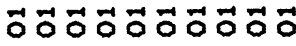

H NOOOOOOOO $0000000-100$

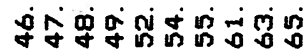




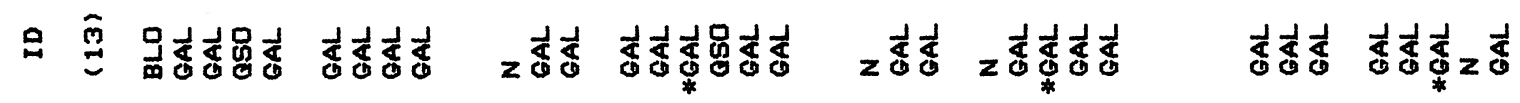

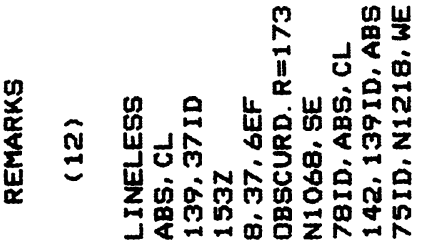

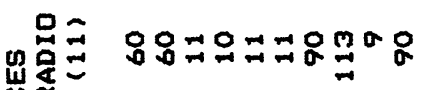

岁远

宸 $N$

㟧品

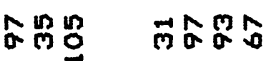

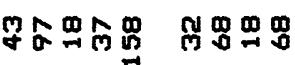

*

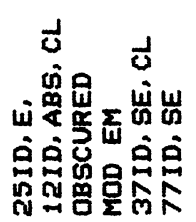

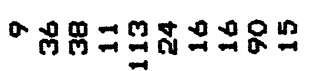

孝守

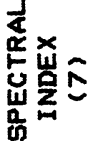

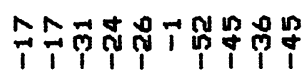

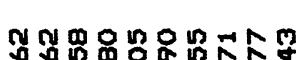

O0000 000000

ú úu

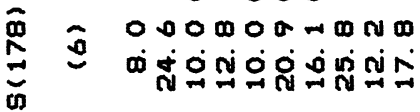

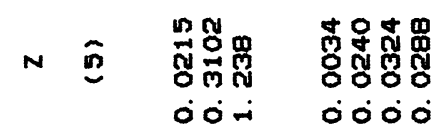

品

虽

岩

号

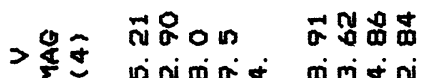

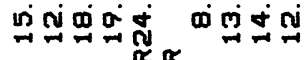

ONAm MONA

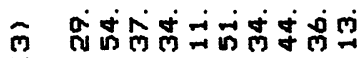

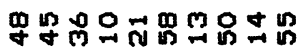

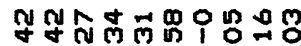

웅

ت

告

高总

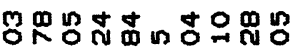

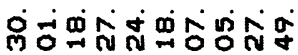

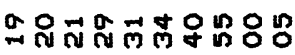

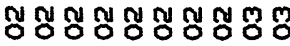

- 0007noOOHo

总 =

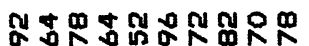

0000000000

' $\quad \begin{aligned} & 1 \\ & 0\end{aligned}$

मOMOMNA+ON

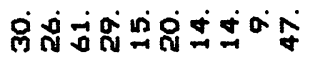

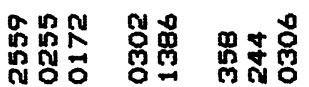

०00 00 000

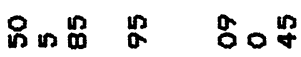

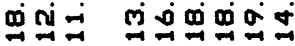

o a OmNNa

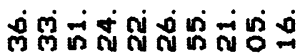

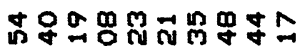

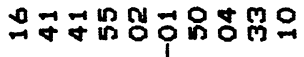

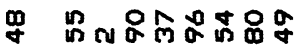

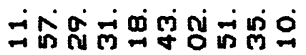

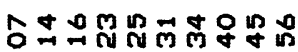

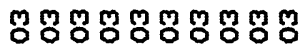

$0-000000-0$

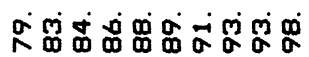

*㟧

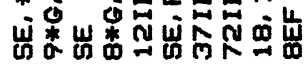

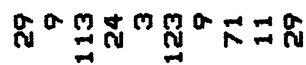

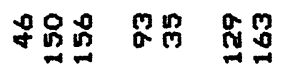

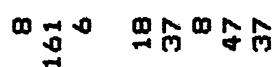

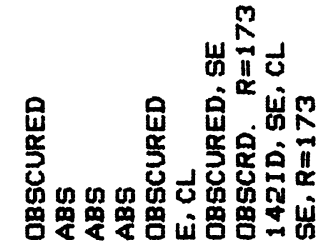

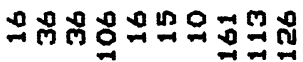

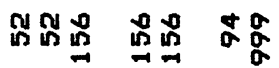

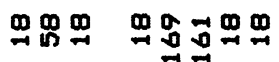

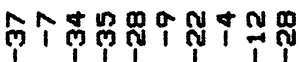

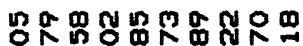

- $00^{\circ}$ - 00000 -

比

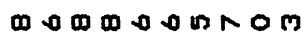

ำ जิ

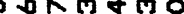

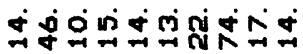

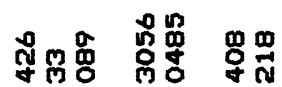

ஸ0ं 0000

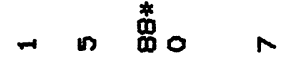

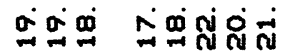

0 ON n

ํำ

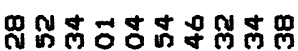

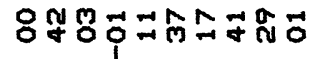

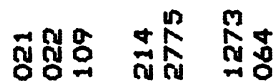
000 00 00

in in 8

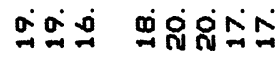

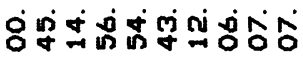

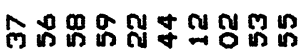

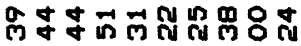

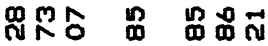

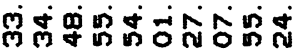

แล大

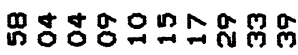

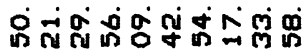

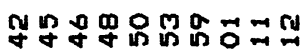

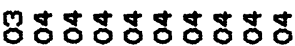

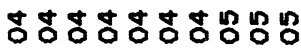

0000000000

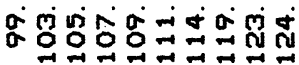

O० ON000000-

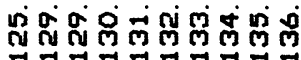




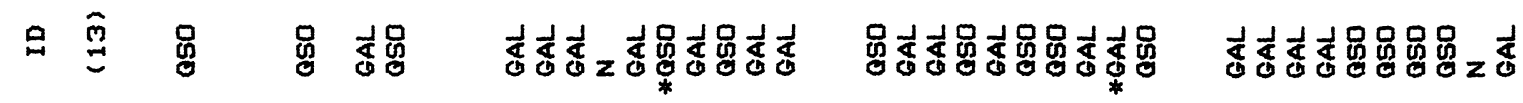

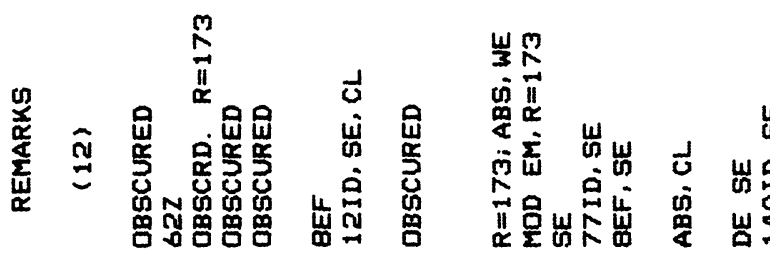

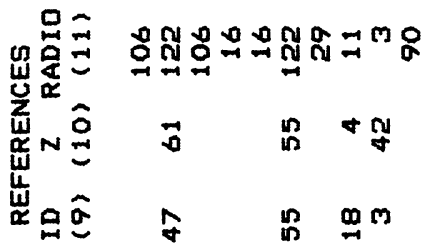

起芯

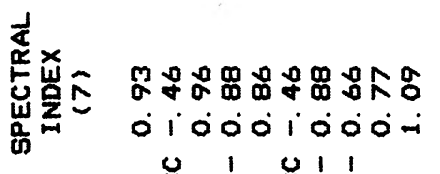
皆 嵌úd

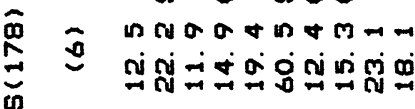

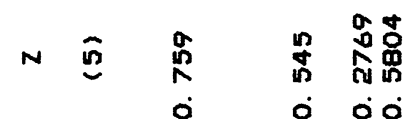

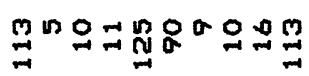

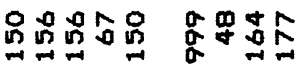

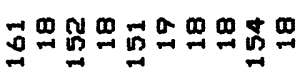

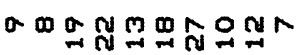

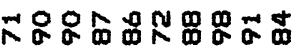
0000000000 Ú m लंष्ब

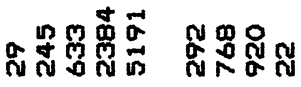
000000000

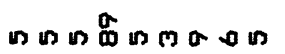

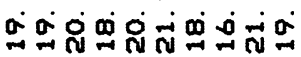

ND OAHDOOH

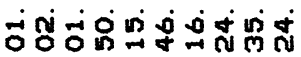

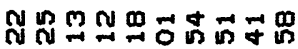

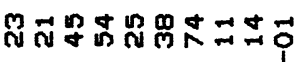

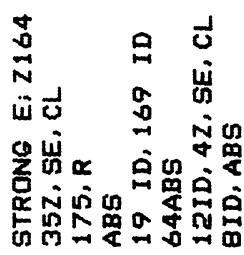

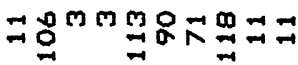

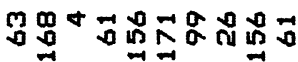

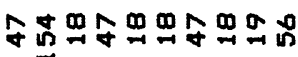

ํำ

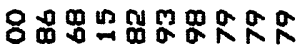
-iórió0.00 '́ ú

แก ก M HOOHA ป

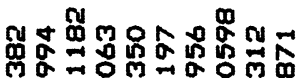
-ió-iorió

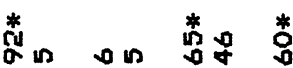

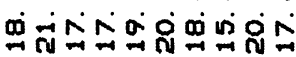

OMmONODOON

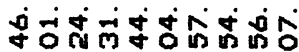

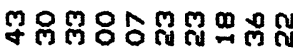

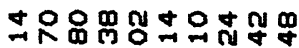

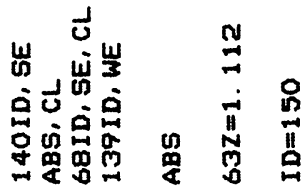

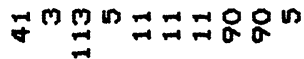

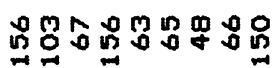

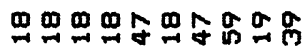

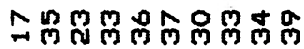

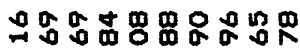
-i00ं ú $\quad+$ ú OHMm ต் ம்

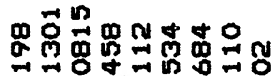

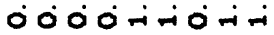

แฺ年

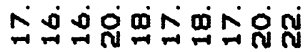

$0,00 \mathrm{datang}$

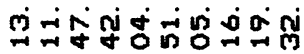

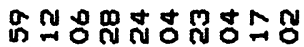

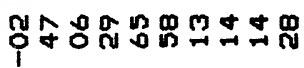

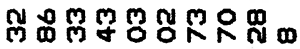

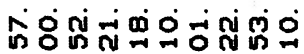

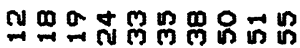

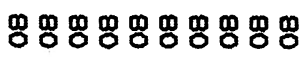

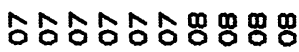
- 000000 मू.

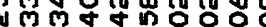

$00-0000000$ 00-000-0 -

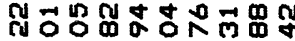

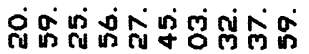
तน

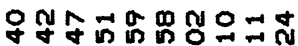
ㅇํㅇㅇํㅇㅇํㅇํㅇㅇํㅇ

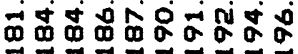




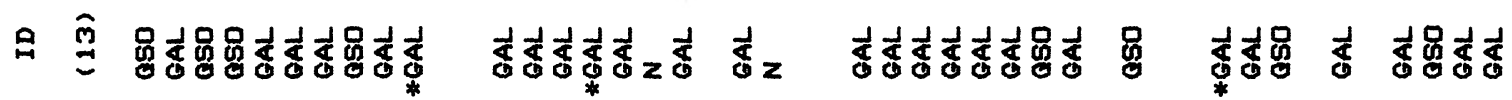

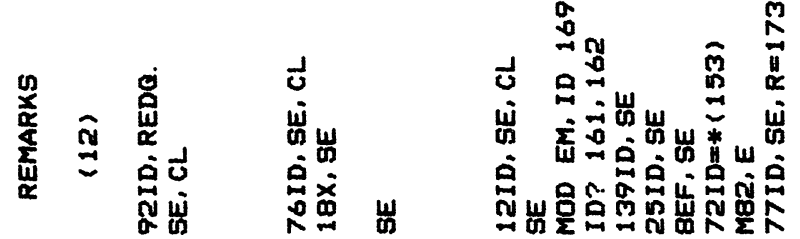

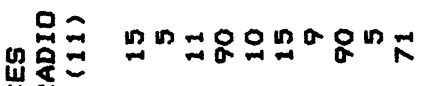

过

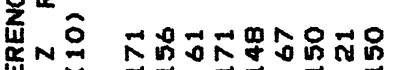

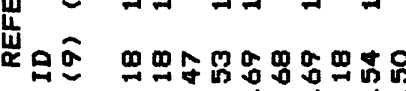

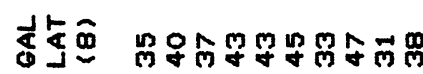

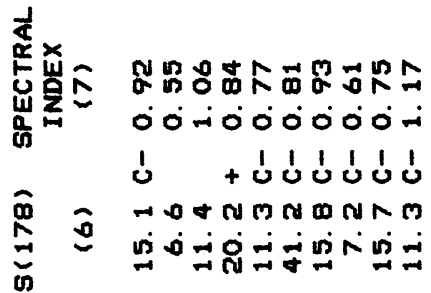

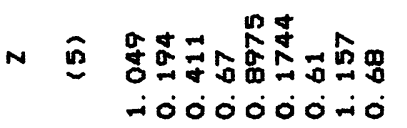

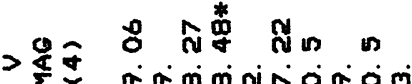

$z_{0}$

๙

$\cos$ Nondon

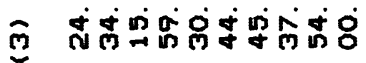

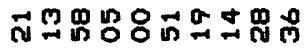

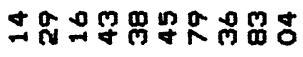

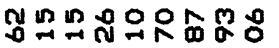

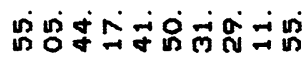

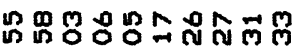

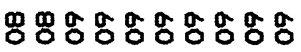

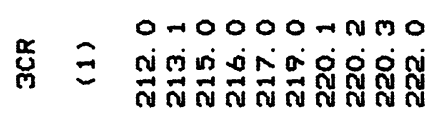

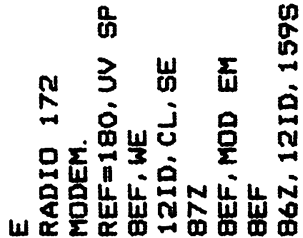

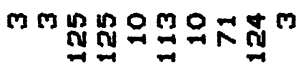

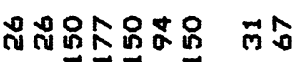

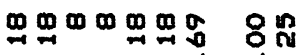

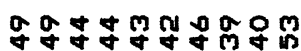

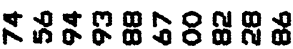

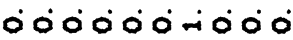

j

ú

nOm oOAmmot

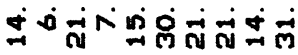

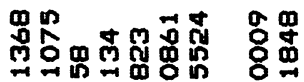

0000000

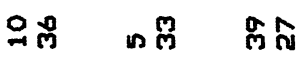

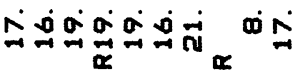

Nanoan

ม่ กู่

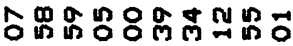

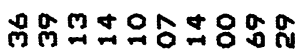

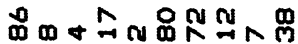

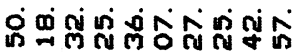

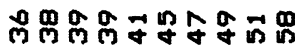

영영영영영영

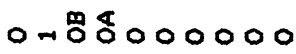

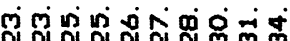

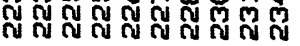

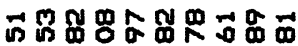

०0.0-00000

لúv

a a namagan

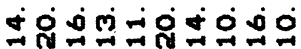

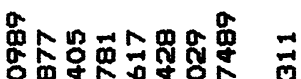

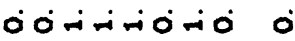

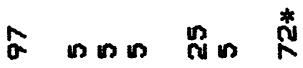

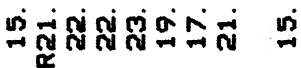

O OndNm-n

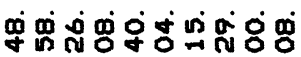

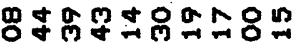

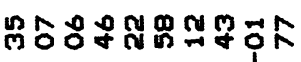

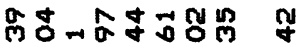
मู่ กิ่

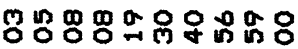

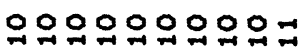

00000 H00

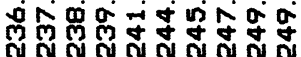

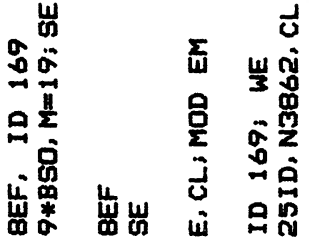

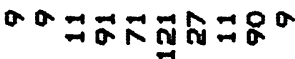

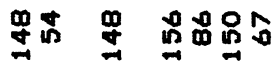

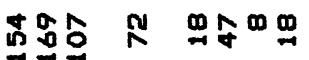

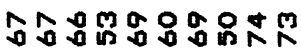

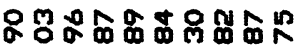

- नio00

d $u^{\prime} d+d$

ómmRN

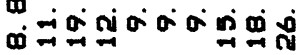

$$
\begin{aligned}
& \text { 옷 } \\
& \text {-i - } 0000
\end{aligned}
$$

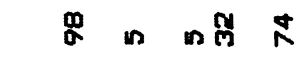

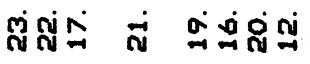

MOO N MOON

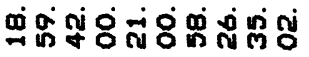

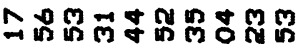

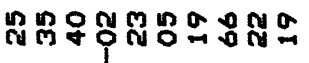

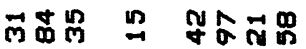

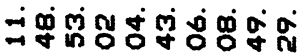

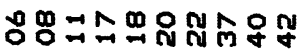

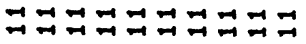

$00000000-0$

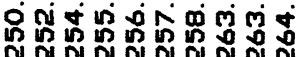




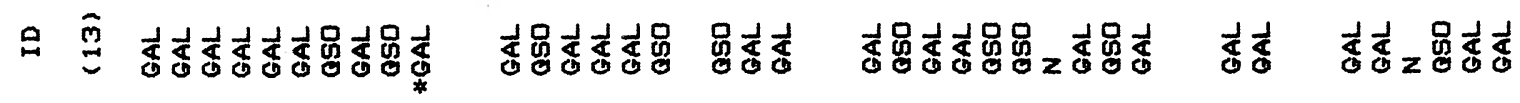

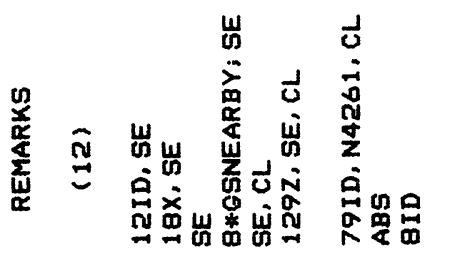

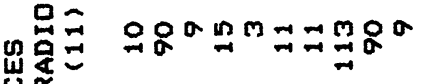

党 $N$

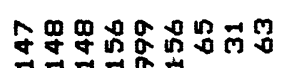

岸品

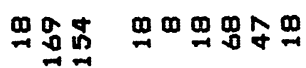

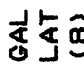

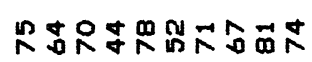

$\frac{1}{\mathbb{x}}$

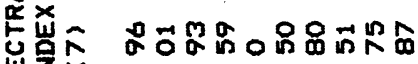

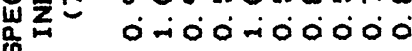

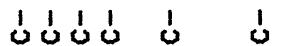

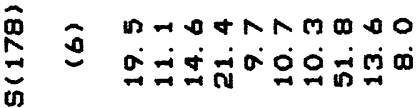

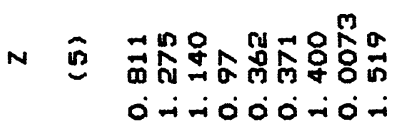

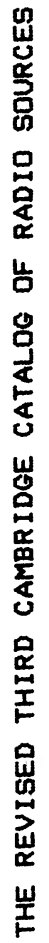

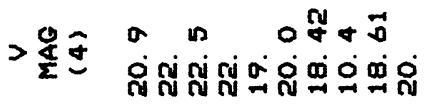

morn nomom

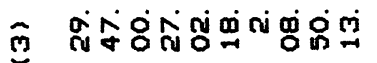

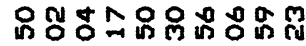

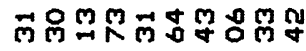

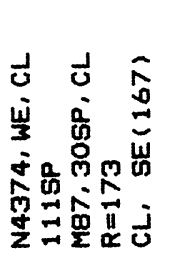

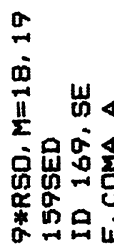

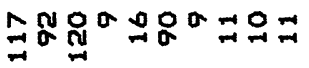

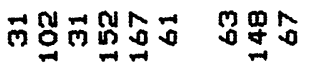

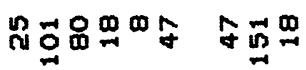

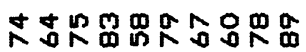

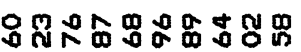
0000000000

u úú ú

+ o vanunoo

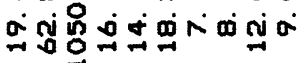

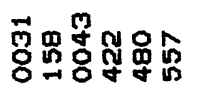

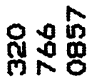

000000 000

ㄴㅎㅇㅇㅛ

$2 \pi$

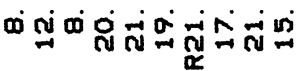

Donmontw

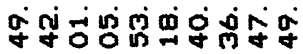

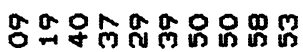

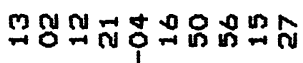

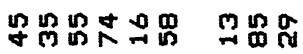

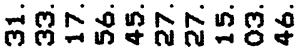

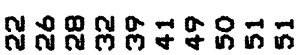

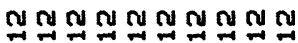

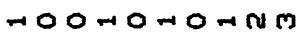

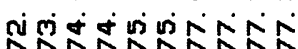

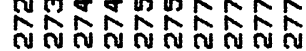

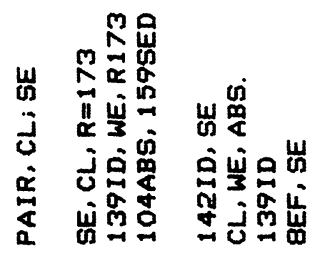

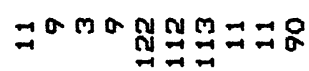

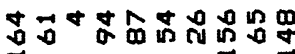

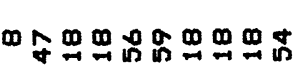

大

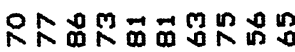

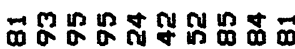

0000000000

1
$\Delta$

N NMOMNOOO

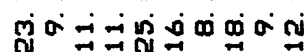

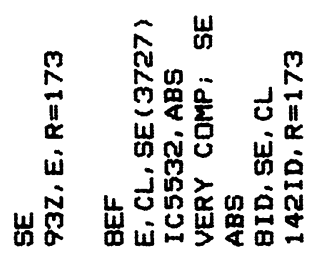

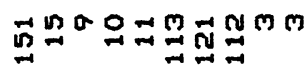

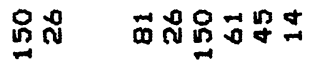

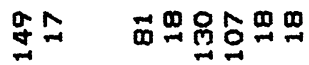

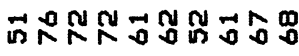

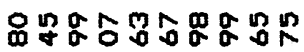

०00

ú ự + ư

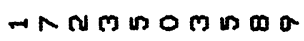

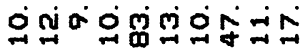

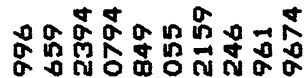

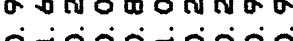

뇽

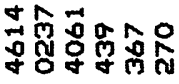

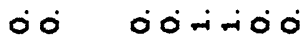

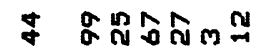

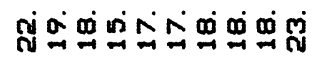

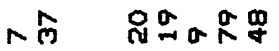

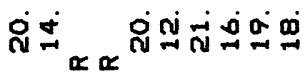

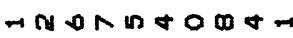

N⿴囗十⺝TH DNT

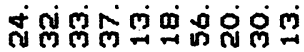

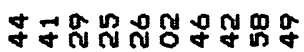

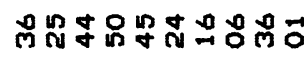

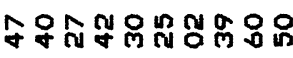

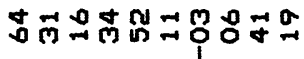

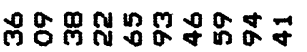

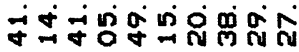

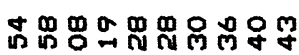
ㄸMMMMMMM

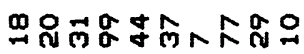

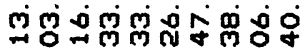

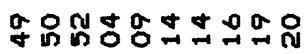
M M M

OHOOOOHOHO

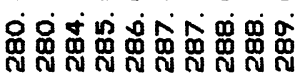

$00-0000000$

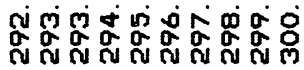




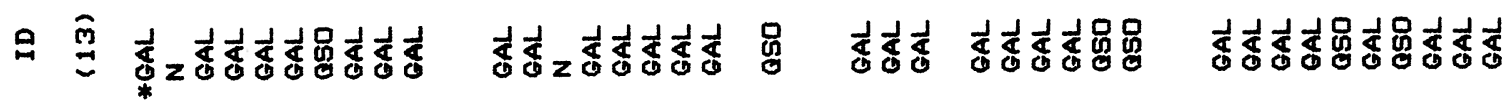

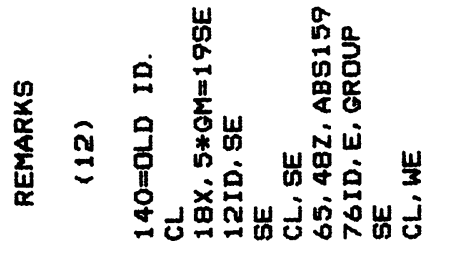

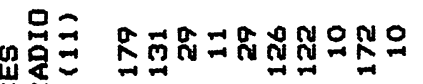

岁这

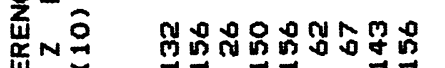

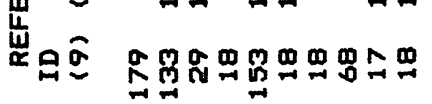

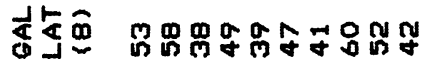

$\frac{1}{\delta}$

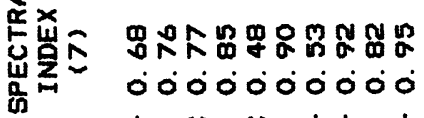

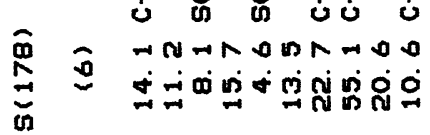

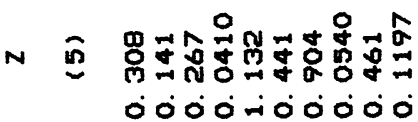

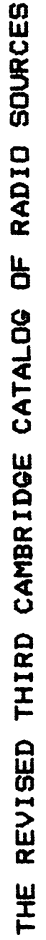

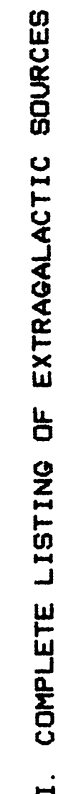

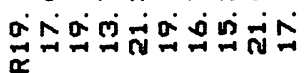

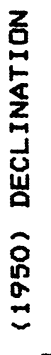

蛋急

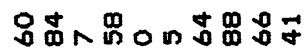

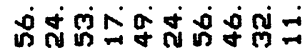

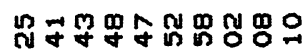

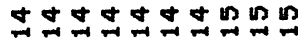

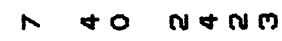

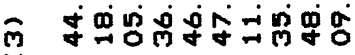

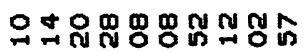

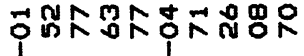

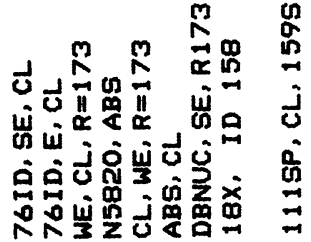

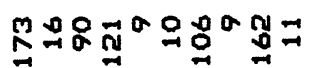

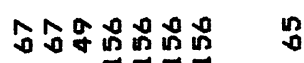

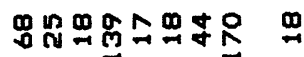

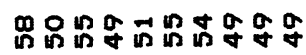

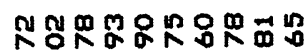

-

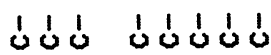

कОMMMन म

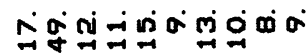

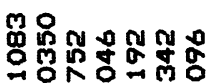

000000

鄵品 n 券

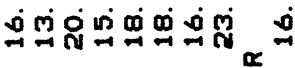

thN tasio in

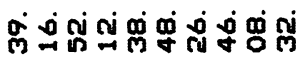

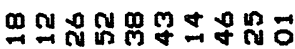

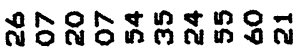

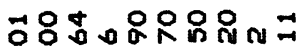

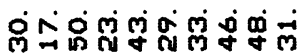

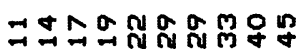

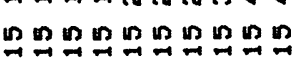

000-100000-

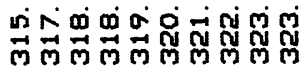

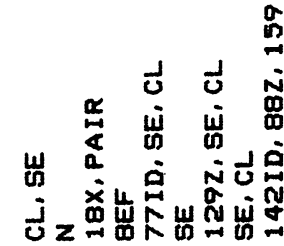

응엉염염

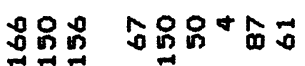

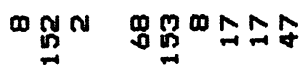

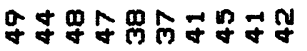

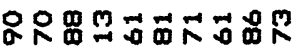
0000000000 úv modmm

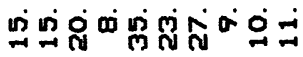

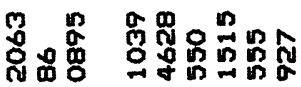
-0் 000000

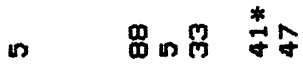

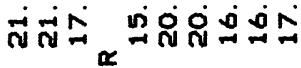

MN ONmono กู่

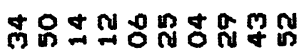

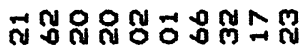

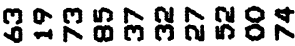
ل́ maーm+m -

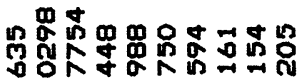
○000000000

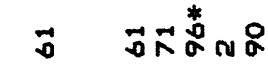

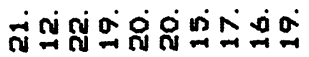

NOO TONNOM

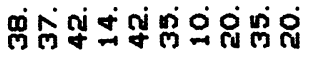

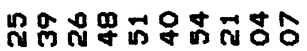

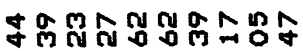

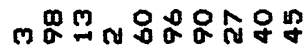

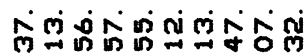

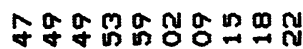

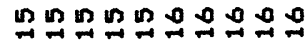

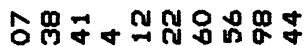
出

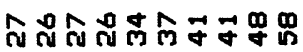

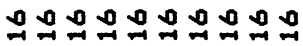

000-0 00000 $00000-0000$

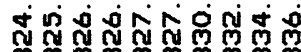

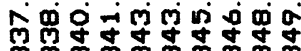




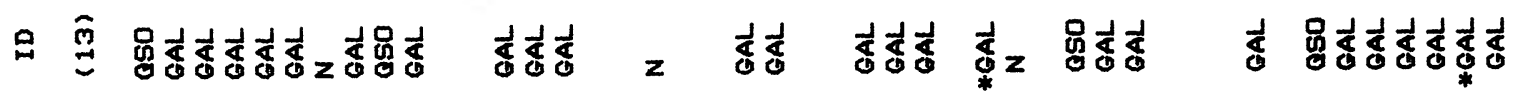

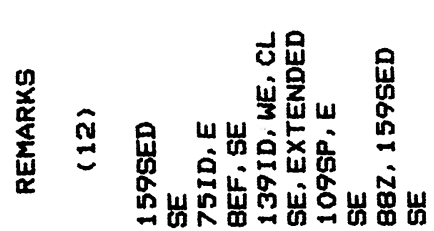

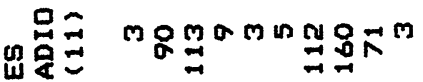

过

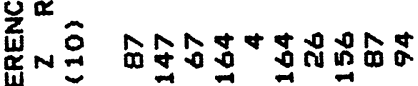

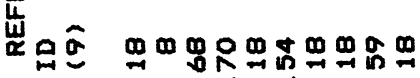

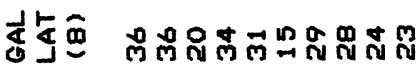

$\frac{1}{\alpha}$

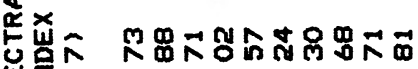

宸证

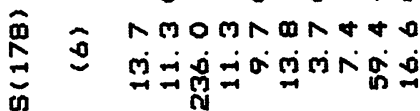

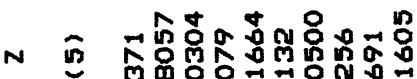

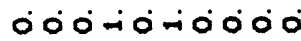

蛡

L

8 d $\quad$ d $\quad x+d$

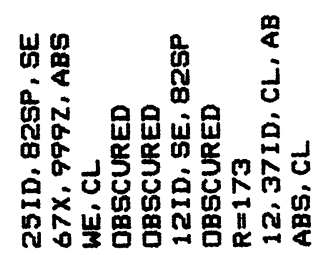

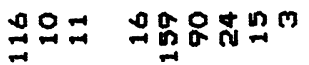

กำ ลี ถับ

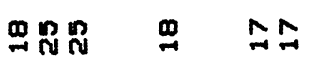

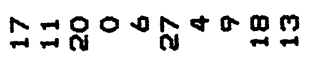

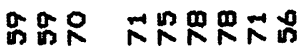
000000000 ú a a 000 mn man

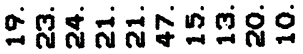

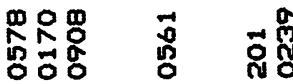
ல0் $\dot{0} 0 \dot{0}$

Mgg

\pm 这

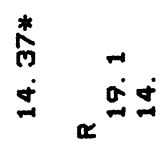

NNN n 0

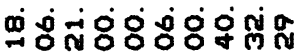

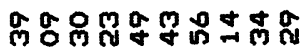

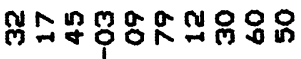

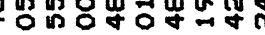

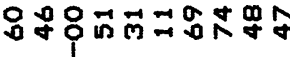
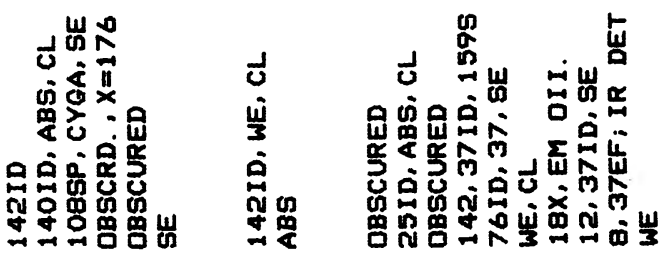

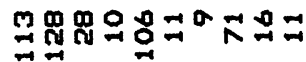

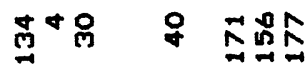

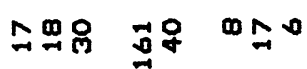

ำ

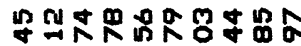

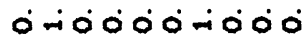
úúd + min. $0000 \% 00$ ำ

点落品

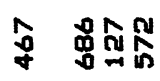
000 $\circ$-100

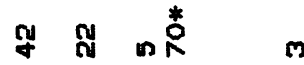

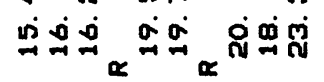

यूत

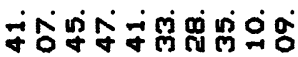

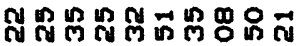

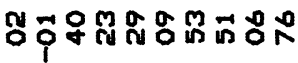

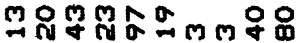

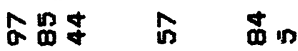

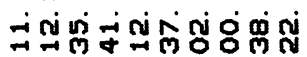

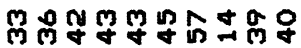

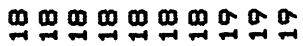

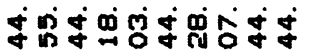

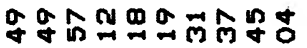
뭄유오ํํํํํำ

$0000000+00$

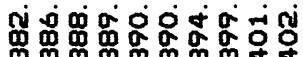

OHOOOONOOH

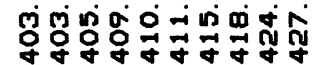

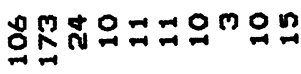

+

思界只品品思思

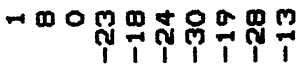

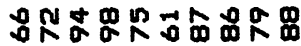
0000000000 ل̛́ú ONMONMOMOR

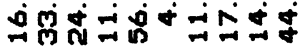

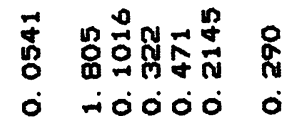

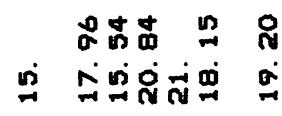

$N+O N m-$

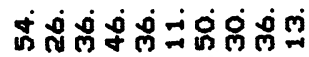

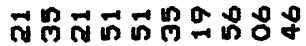

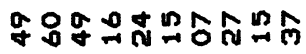

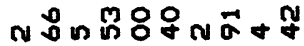
भुष

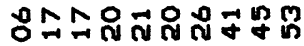
ลีลี สีกีกีสีลีสีสี

0000000000

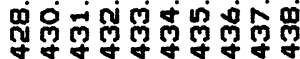




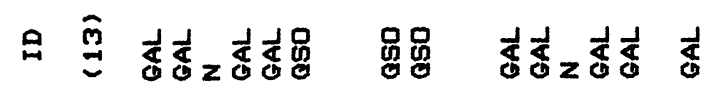

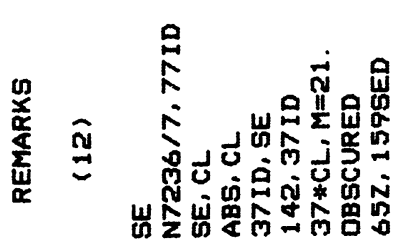

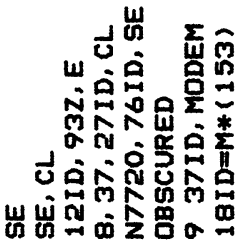

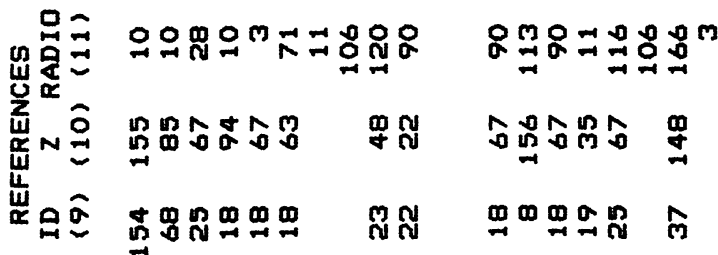

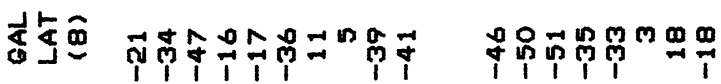

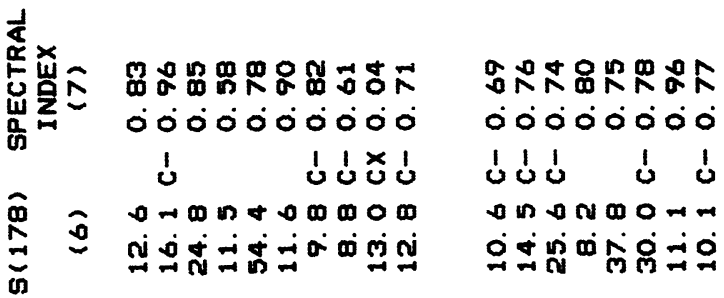

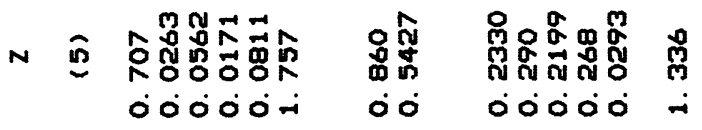

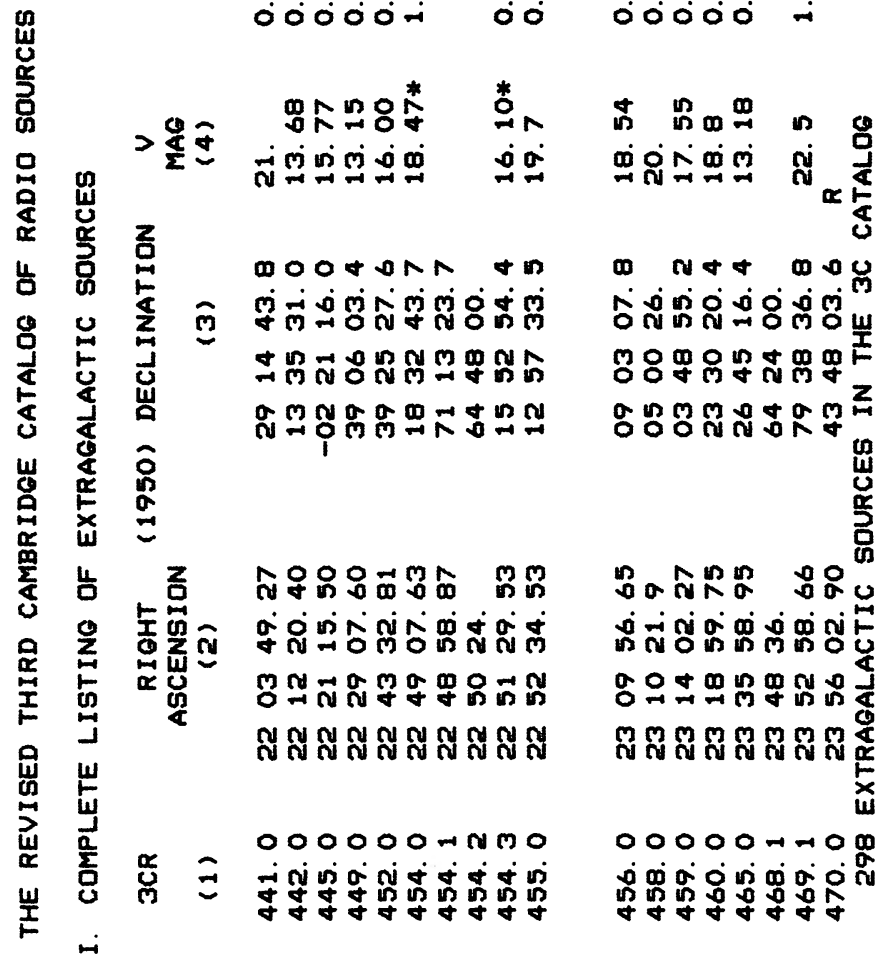




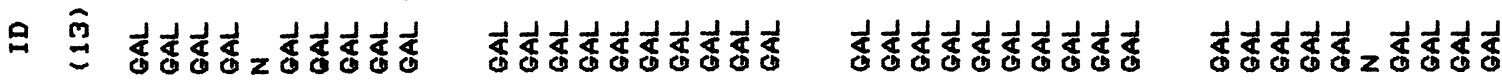

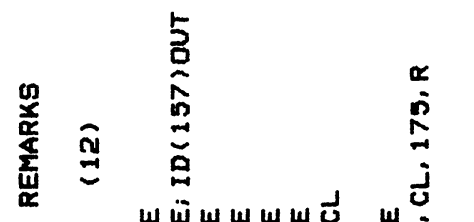

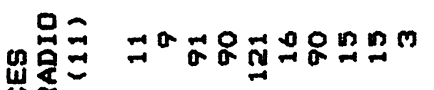

妾

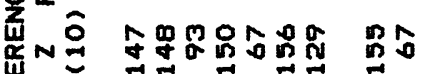

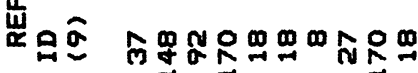

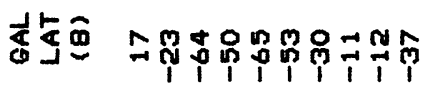

$\frac{1}{c}$

点剻

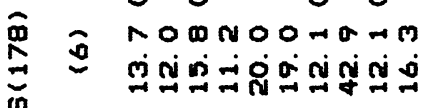

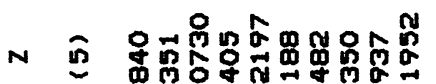
Oـ 00000000

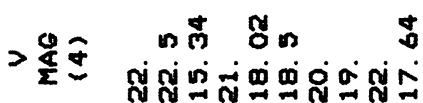

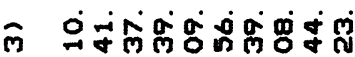

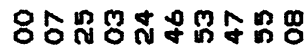

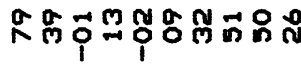

岩

品

品

$\stackrel{m}{\stackrel{m}{x}}$

똔

८৪८:8৪8:8:

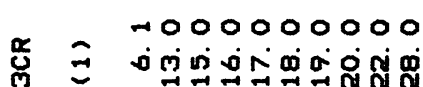

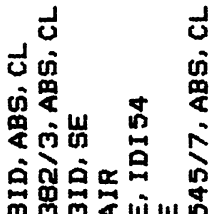

要 岇㟧

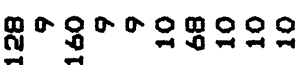

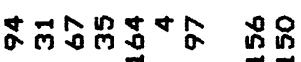

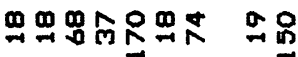

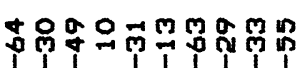

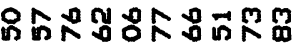

०000-ió0.0

$$
\text { ú }
$$

u

motonasona

iिं

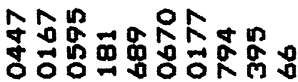
0000000000

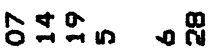

ปึ่

amatammona

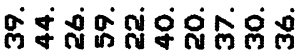

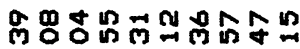

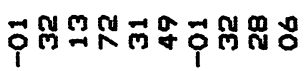

触男思品每合

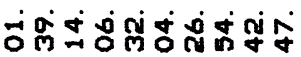

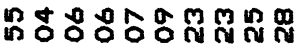

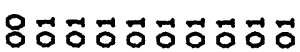

$000-000000$

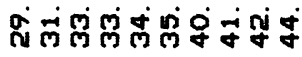

岗

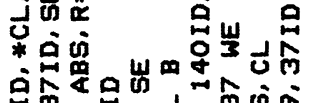

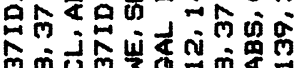

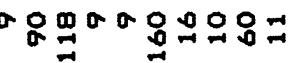

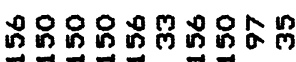

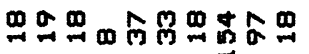

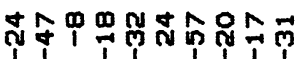

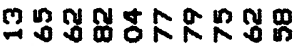

-i000

Ú

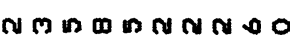

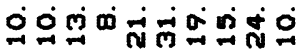

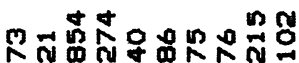

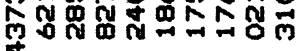
०.00000000

n i mon

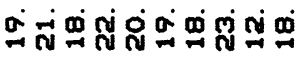

on mu mo

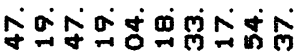

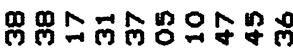

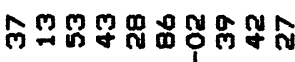

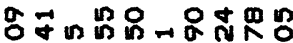

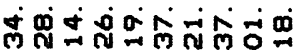

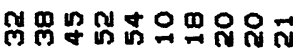

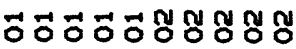

$00000=0000$

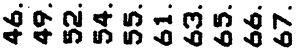

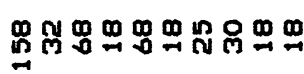

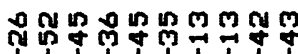

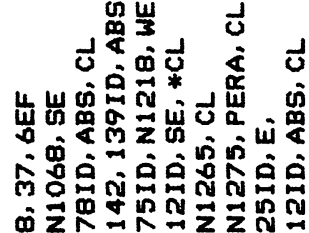

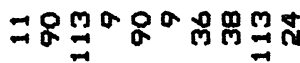

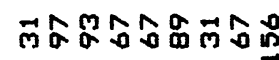

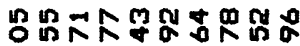
-ió0000000 أ

OM

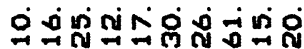

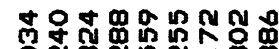

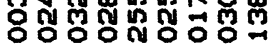
000000000

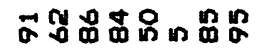

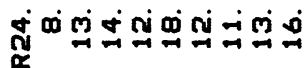

NMOHनO\&OOM

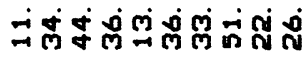

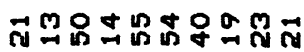

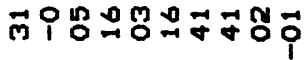

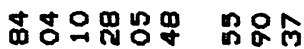

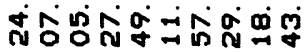

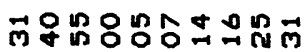

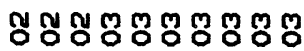

NOOMOOHOOO

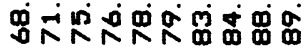




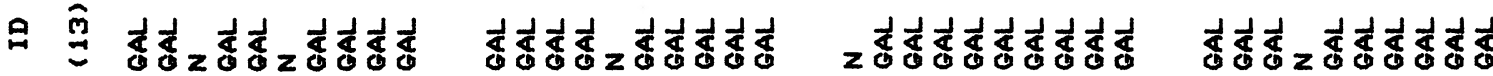

竞

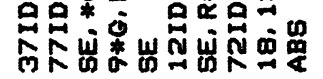

品是=

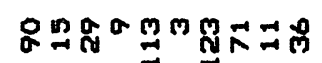

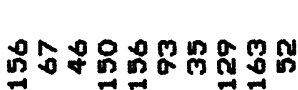

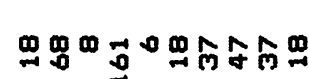

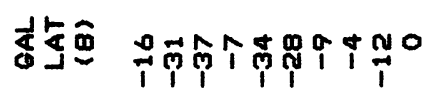

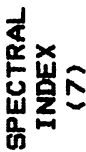

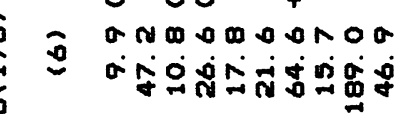

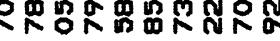
$00^{\circ} 0^{\circ} 0^{\circ} 0^{\circ} 0^{\circ} 0^{\circ}$ ป

in

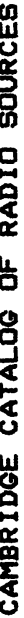

N ล 0000000000

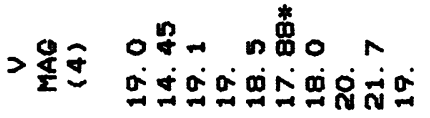

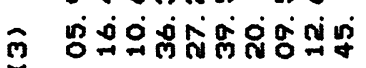

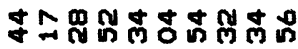

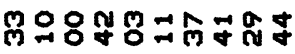

品

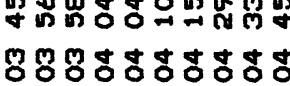

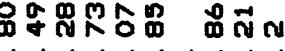

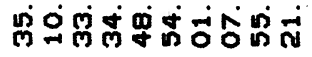

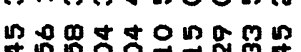

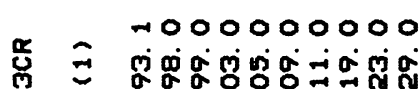
엉워
登

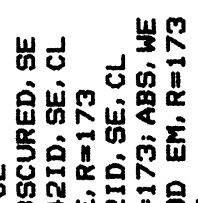

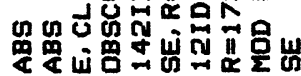

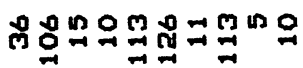

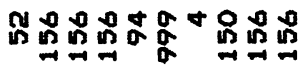

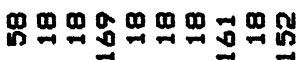

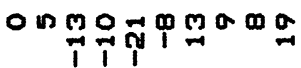

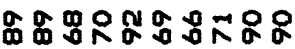
0.0000000 út

MINMmOMUNm

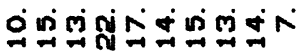

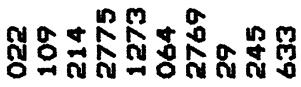
0000000000

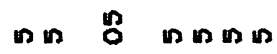

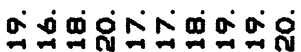

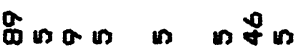

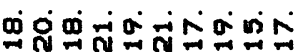

0400 thmona

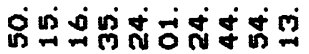

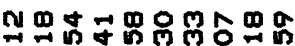

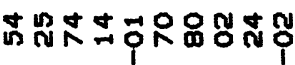

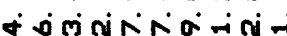

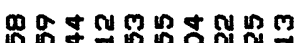

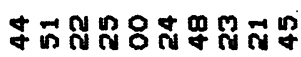

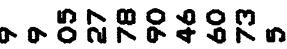

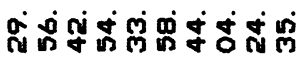

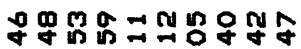

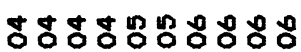

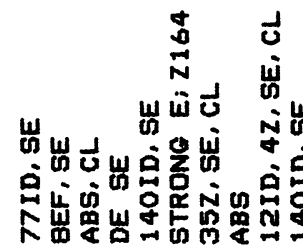

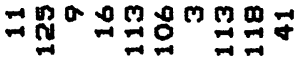

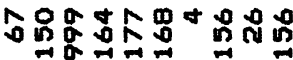

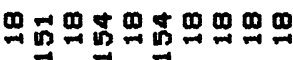

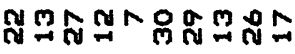

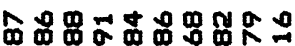
000000000 . ป่́

undadanoman

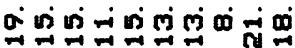

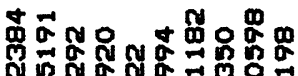
0000000000

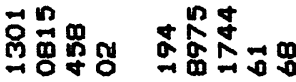
000न 00000

is $\mathbb{R}$

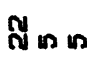

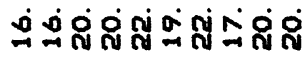

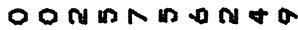

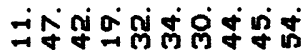

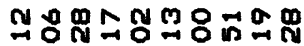

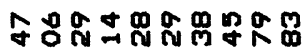

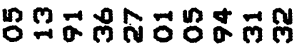

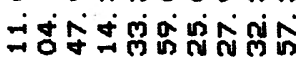

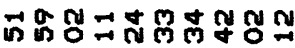

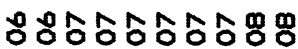

品品哭思

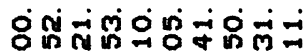

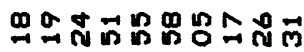

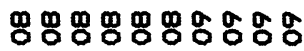

m0000-000-

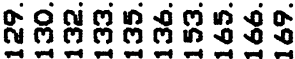

OOH-OOHOOH

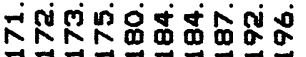

HOOHOHOOHM

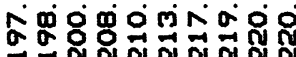




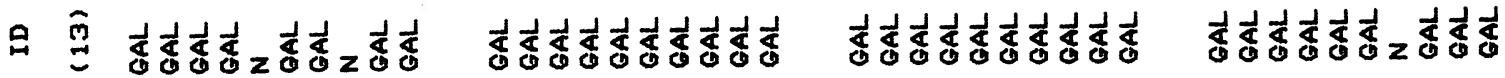

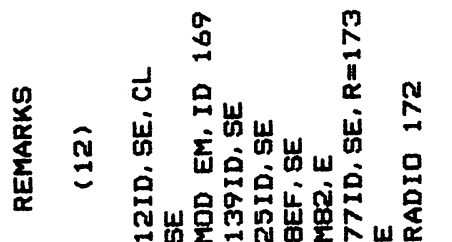

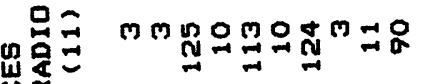

运

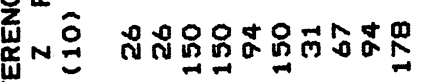

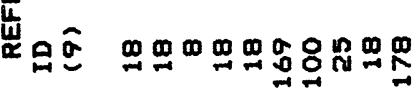

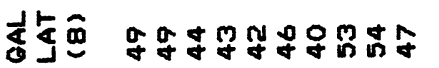

$\frac{1}{d}$

点䓛完

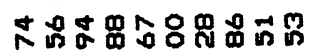

00000 -io000

ป́

क a nomoronata

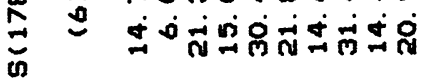

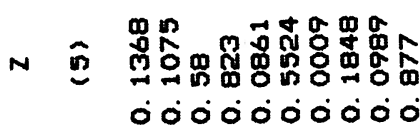

㞻
总
品
品
定 กำ

品

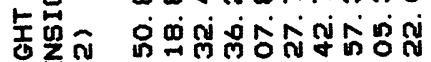
造

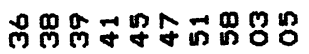

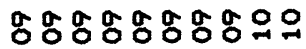

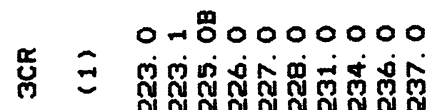

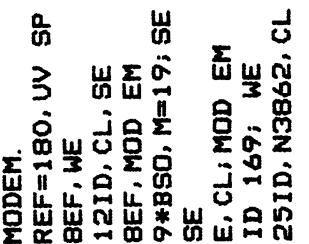

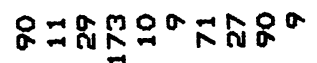

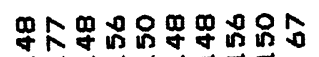

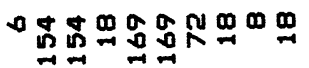

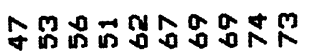

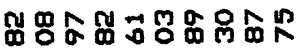

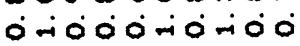

úúú

OnOmDOMnNo

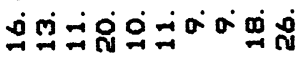

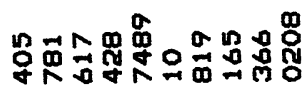

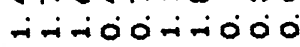

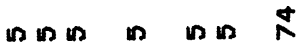

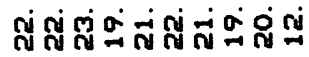

UANMUnNOAN

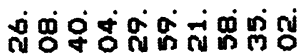

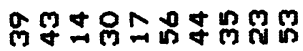

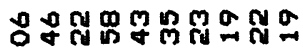

z

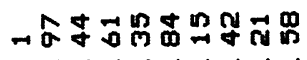

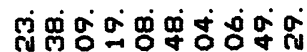

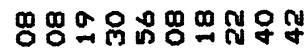

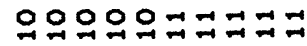

000-0000-0

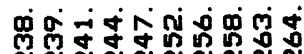

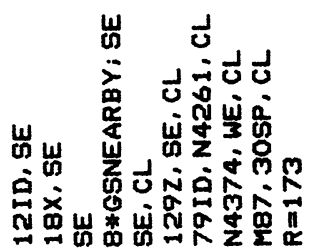

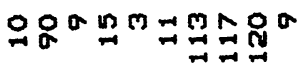

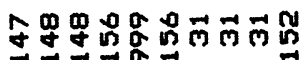

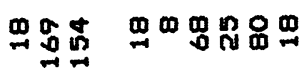

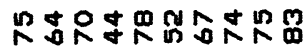

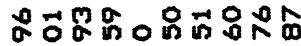
O-100-100000 ป́u์ú ú n- 0 annotim

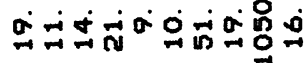

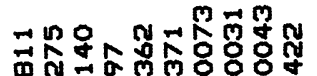
O-

- $200 \hat{0}$

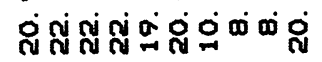

-

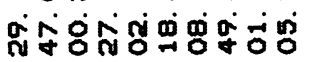

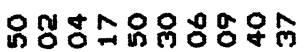

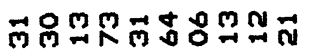

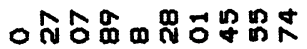

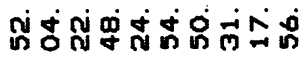

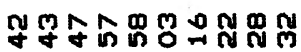

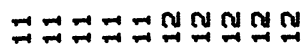

OOOHNMOHOH म่

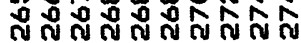

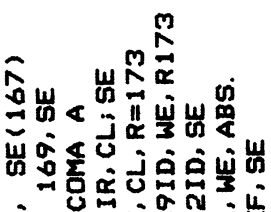

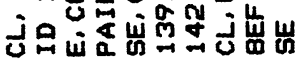

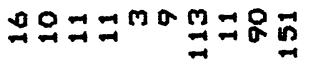

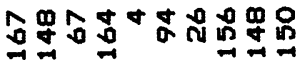

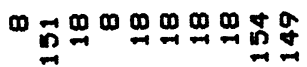

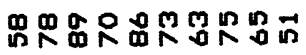

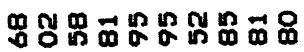
oriojosotó d $\quad$ u + ú nOONMMNOON

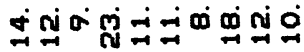

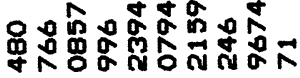
0000000000

कू बूरू ลี่ ถี่

amin-ONODM

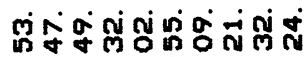

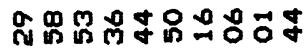

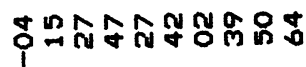

ㅇำ 品กิ

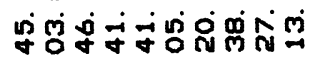

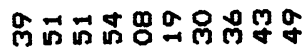

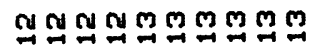

ONMOOOHOOO

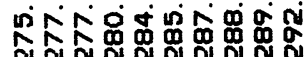




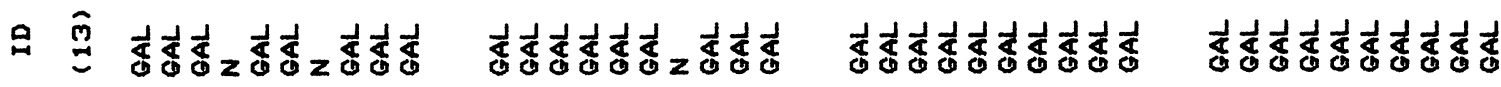

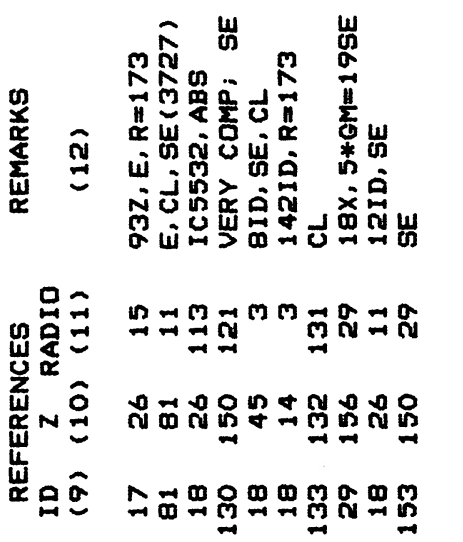

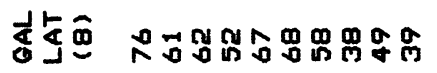

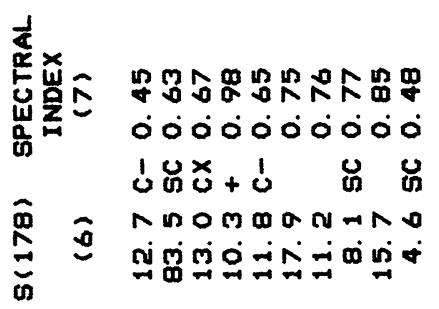

$N$ ล

ปถ่

mon man to

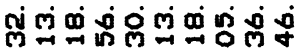

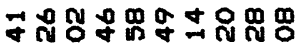

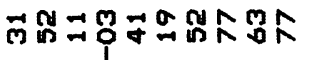

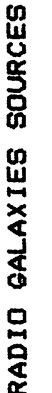

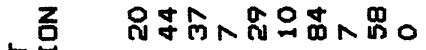

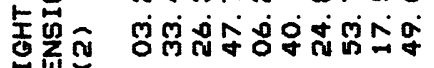

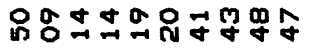

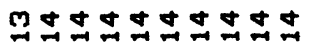

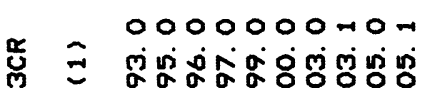

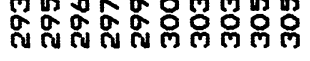

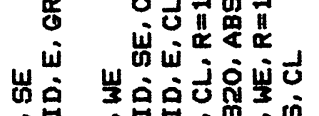

$\ddot{m}$
高

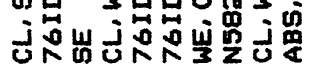

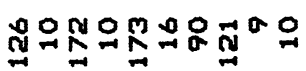

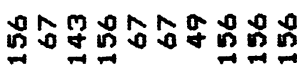

思罟吕罟员思总邑思

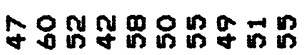

여ำ

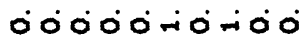

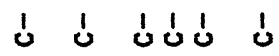

In $0000 \mathrm{mmm}$

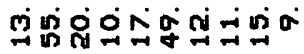

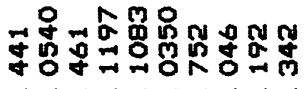
○ं0் 000000

창ํำ in

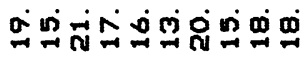

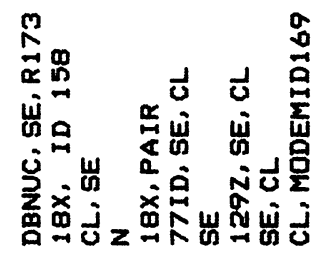

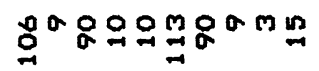

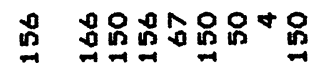

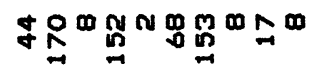

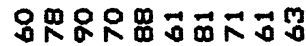
000000000 úúcúc m

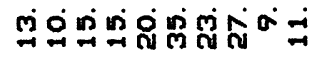

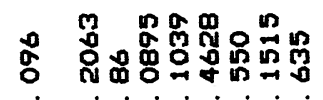

anm+nR $2 \pi$ คั

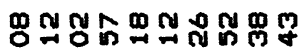

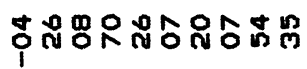

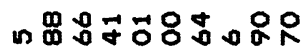

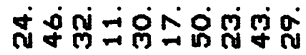
ญูญ์

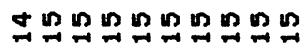

- $00-000-00$

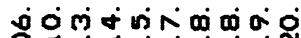

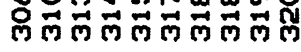

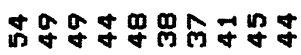
- rio00000

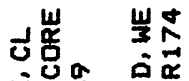

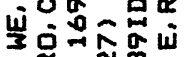

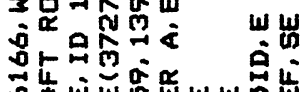

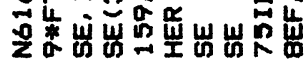

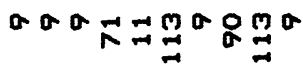

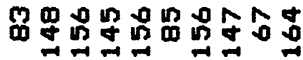

只品界思思四罗足

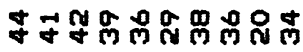

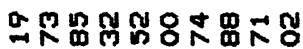
-0000 00000 ل́ a m n I O MOM

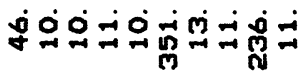

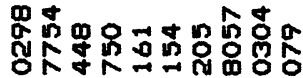
000000000 -

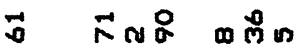

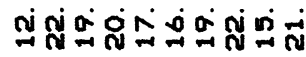

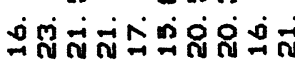

nO MNONMON ถิ่

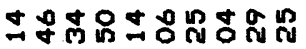

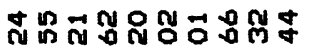

ON ONOMOMN

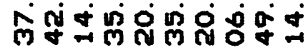

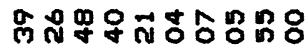

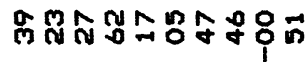

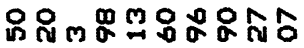

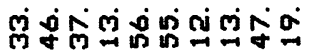

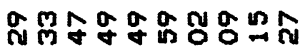

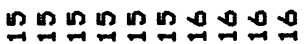

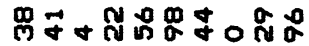

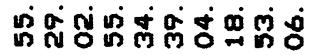

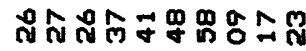

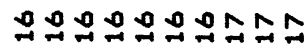

$000000-000$ $000-000000$

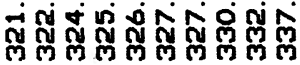

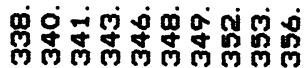




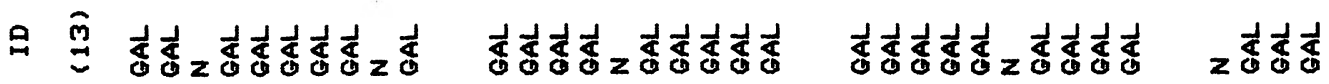

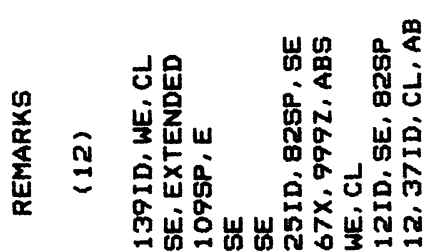

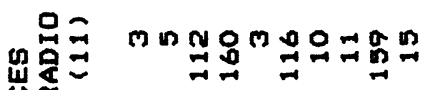

岁安

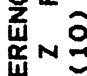

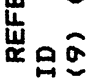

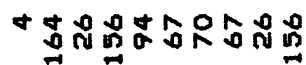

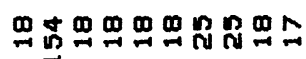

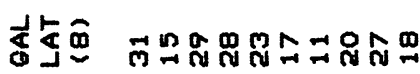

$\frac{1}{\alpha}$

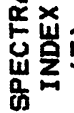

品

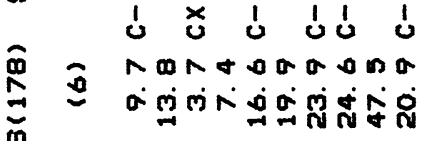

N ล

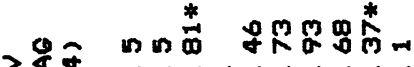
में

ว กู่

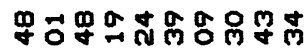

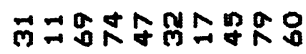

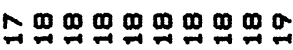

- 00070000mo

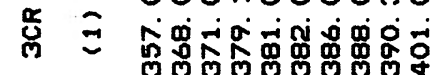

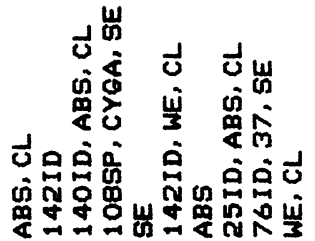

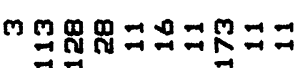

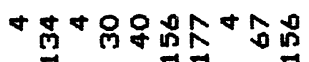

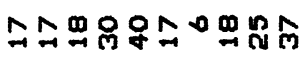

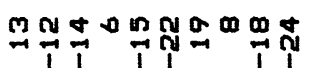

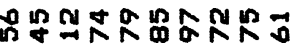

○ं

ú $\quad$ ú $\quad$ ú

T西的.

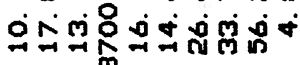

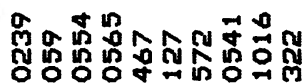
0000000000

ช สู้ำ $m$ แั้

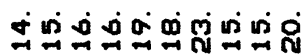

IN MUNARON

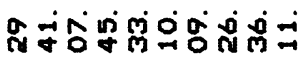

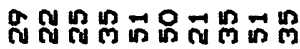

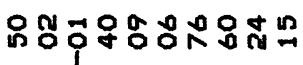

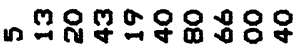

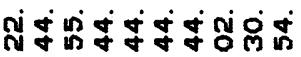

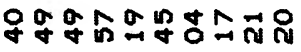
๙

00-000-000
㟔㟧

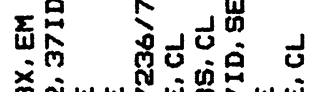

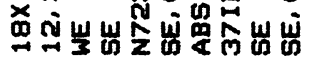

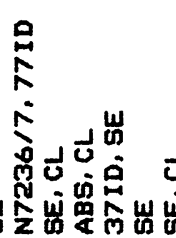

宸竞

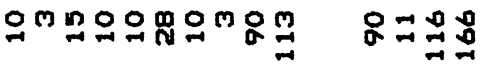

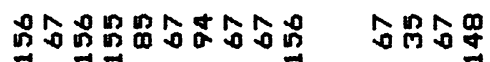

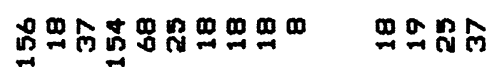

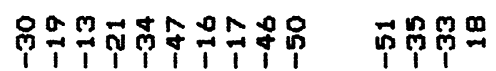

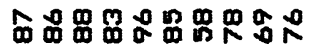

0000000000 ú ú ú omnom om

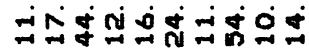

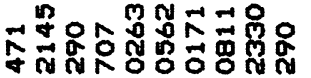
0000000000

สู ผูํํำ 0ं०

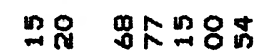

तं क्ष

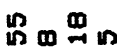

ำ

$m \rightarrow \infty 00+00$

c4t+m

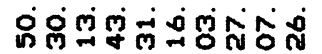

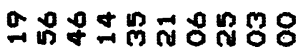

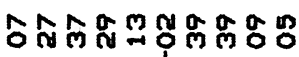

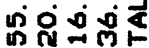

品思

ณำกำ

๙ กิกำ

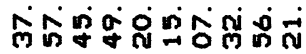

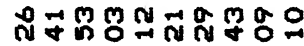
ลี สี สีกํํํํำ

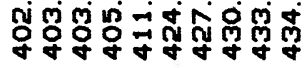

0000000000

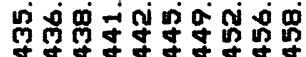

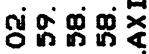

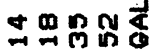

ชูำกำ

2.07

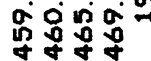


0

을

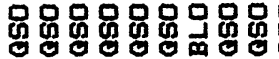

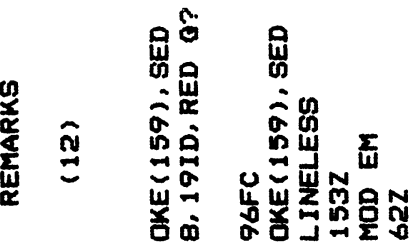

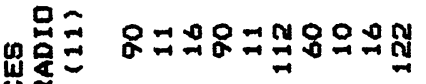

可

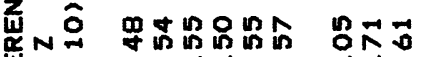

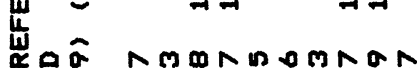

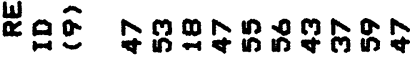

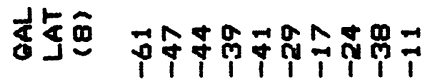

$\frac{1}{4}$

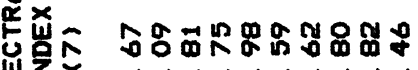

㟧

J U Ju

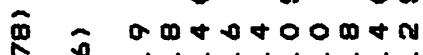

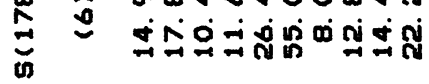

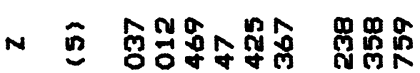

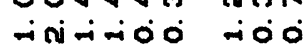

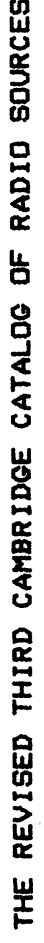

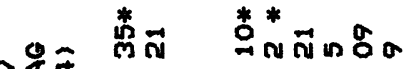

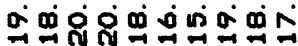

z

(1)

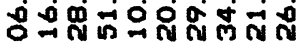

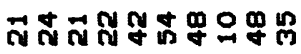

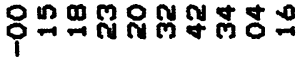

量

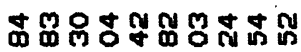

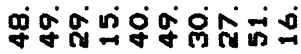

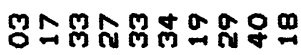

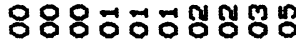

$0000000-00$

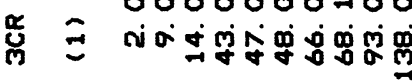

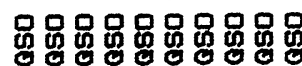

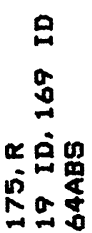

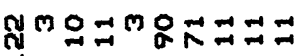

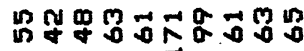

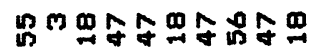

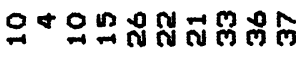

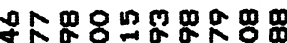

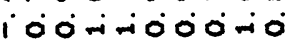
iั

nntannoond

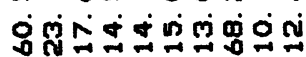

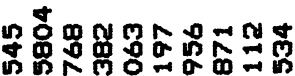

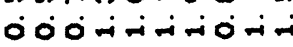

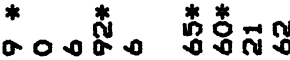
ํำ

m 00000hat

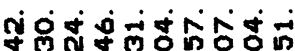

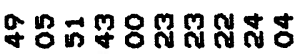

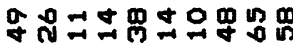

品品品品品品品品品品

品品品品品品品品品品

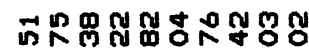

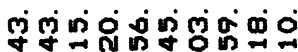

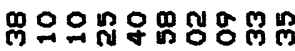

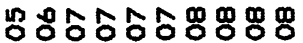

0000000000

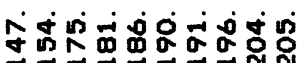

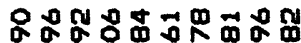
0ं0ंग0ं0000 + ú + ú ommanchan लिख्न

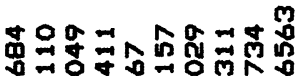

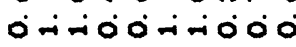

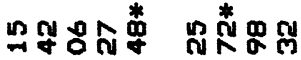

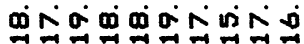

+onnoomoo

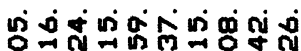

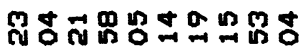

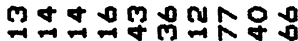

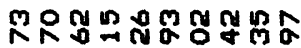

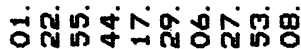

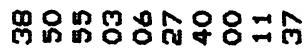

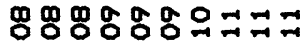

OOOOONONOO

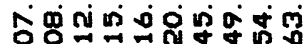

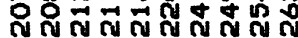

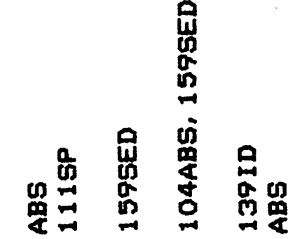

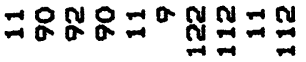

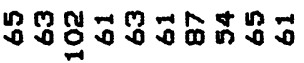

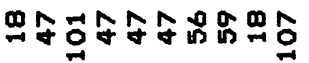

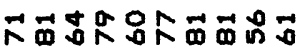

ำำ 0000000000

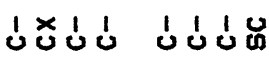
momm nNOMOM ำ भु่

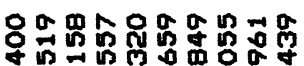

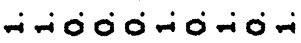

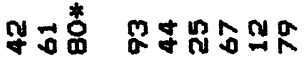

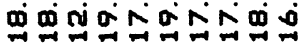

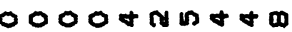

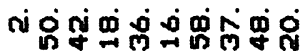

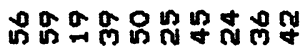

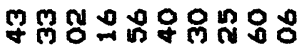

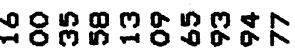

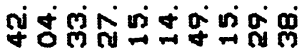

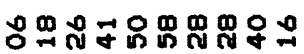

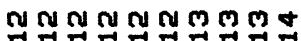

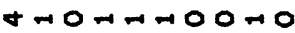
ตำ กีลลิล ลิ 


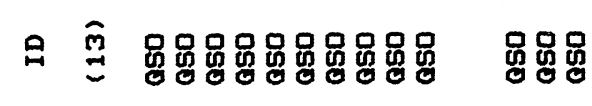

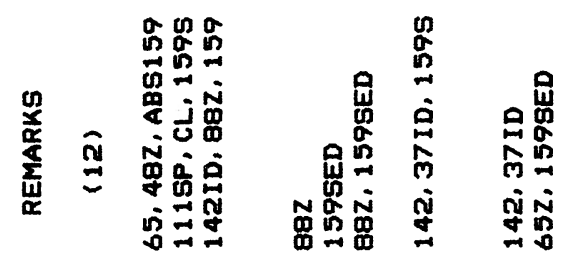

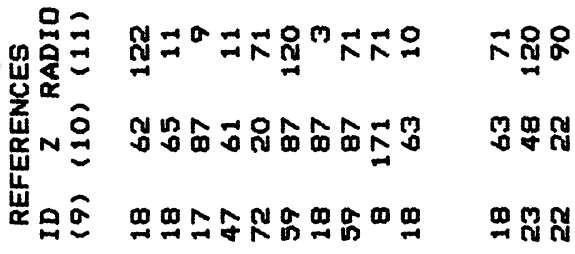

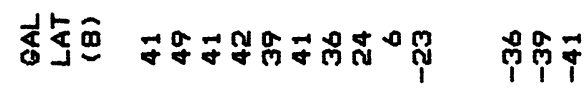

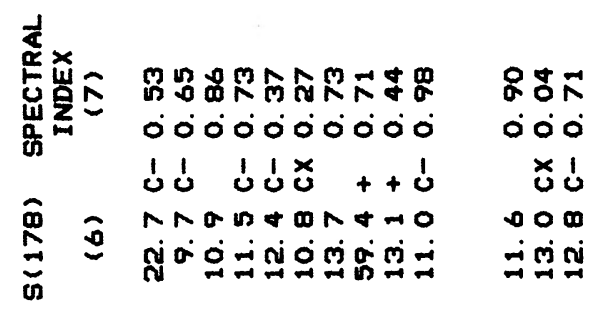

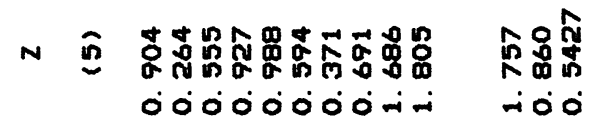

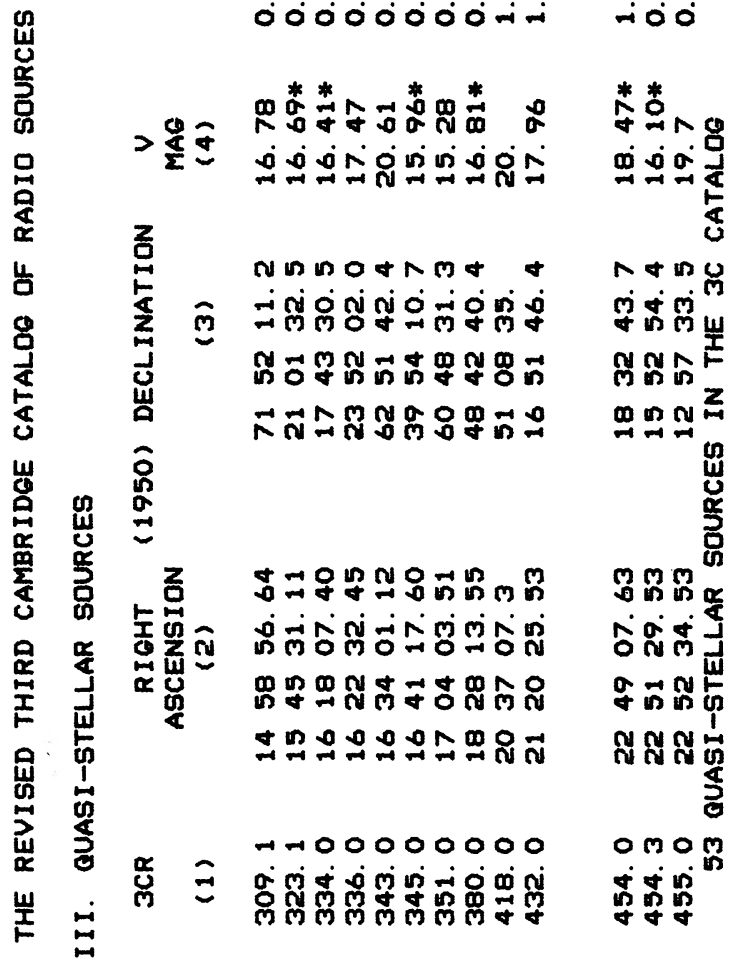




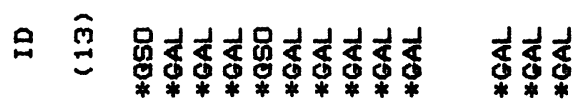

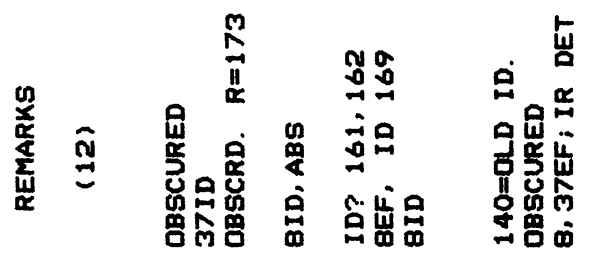

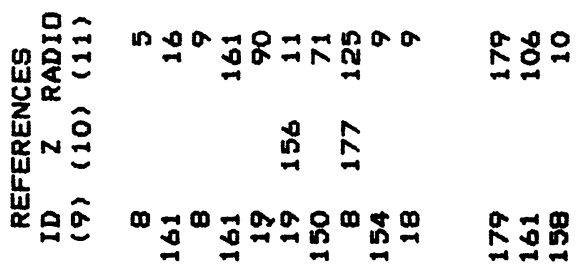

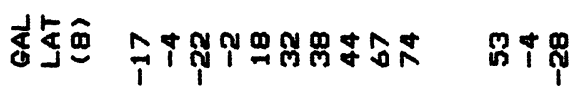

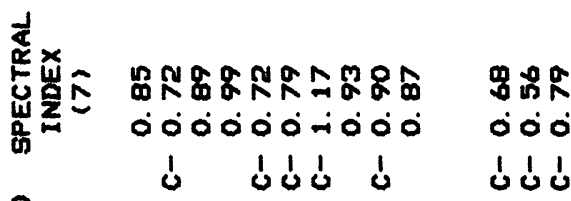

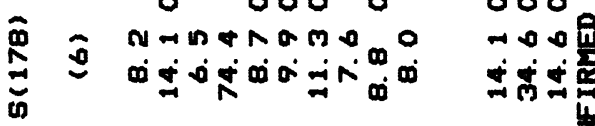

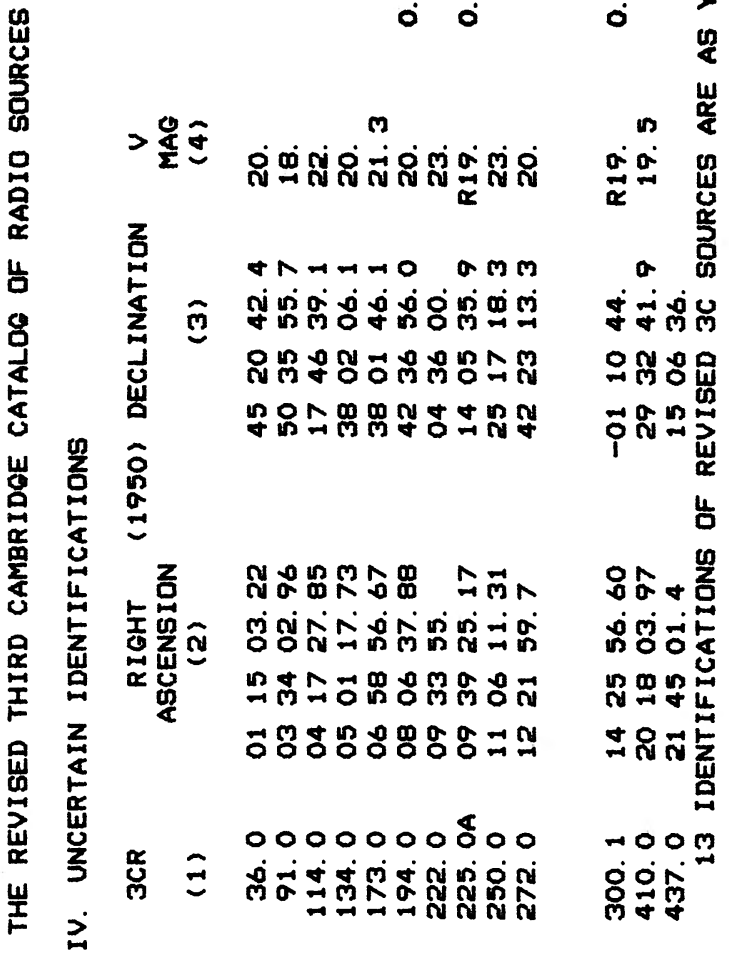


욜

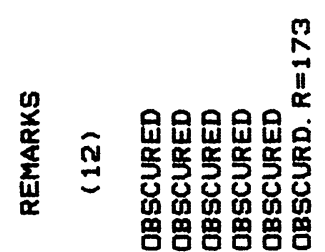

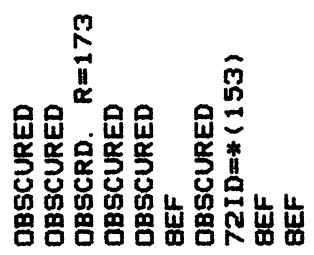

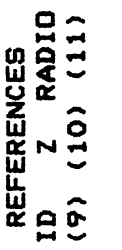

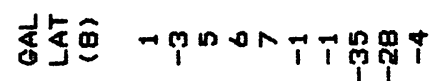

卖产出

离

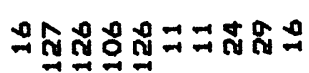

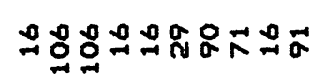

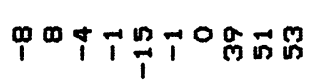

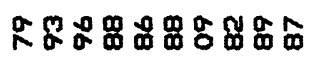
0000000000 Ú d d d d d t monno omm-

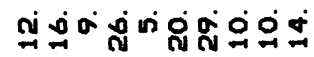

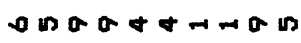

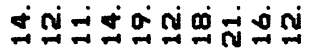

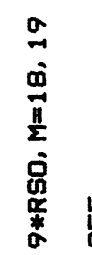

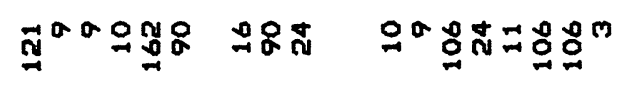

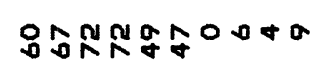

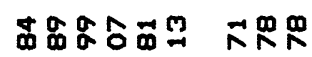
Dótió 000 ú N BnMtroOm

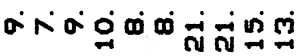

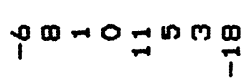

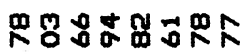
O- 0000000 ป Uúúú

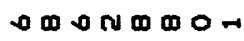

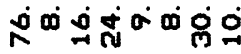

N

怘

告

兽

$>\frac{8}{2} \hat{y}$

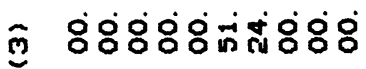

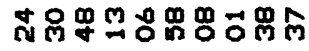

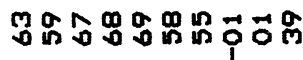

a

m

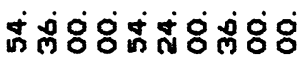

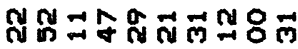

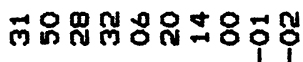

용

要

\section{氛总}

in

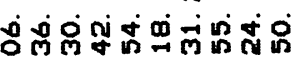

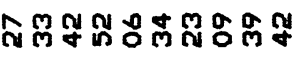

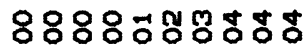

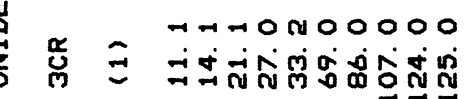

ดमmMO N

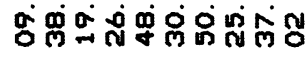

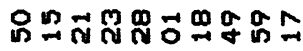

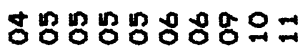

OONO-0000O

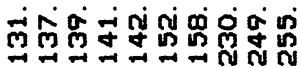

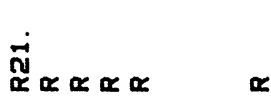

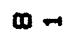

ํำ

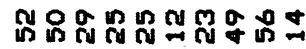

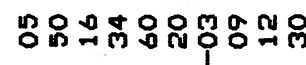

ตัด् ณ

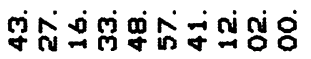

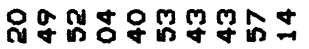

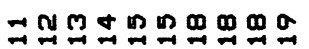

00-00-000-

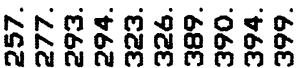

$\propto \propto$

no 0 o

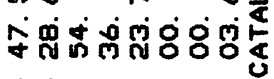

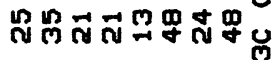

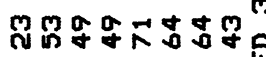

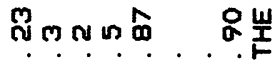

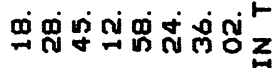

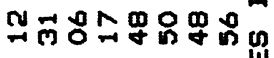

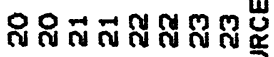

ONOONNTO

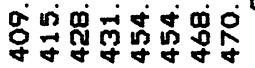




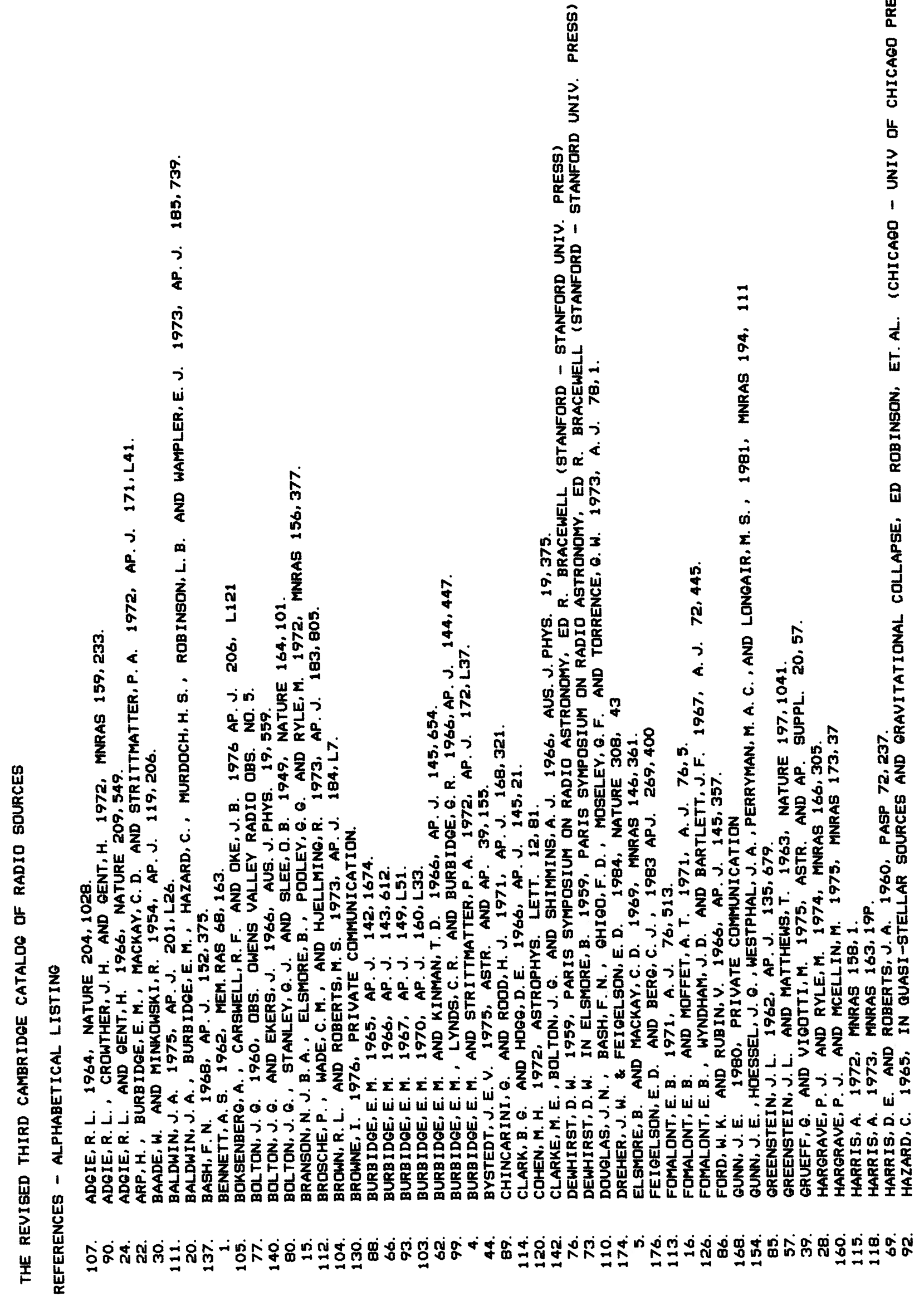




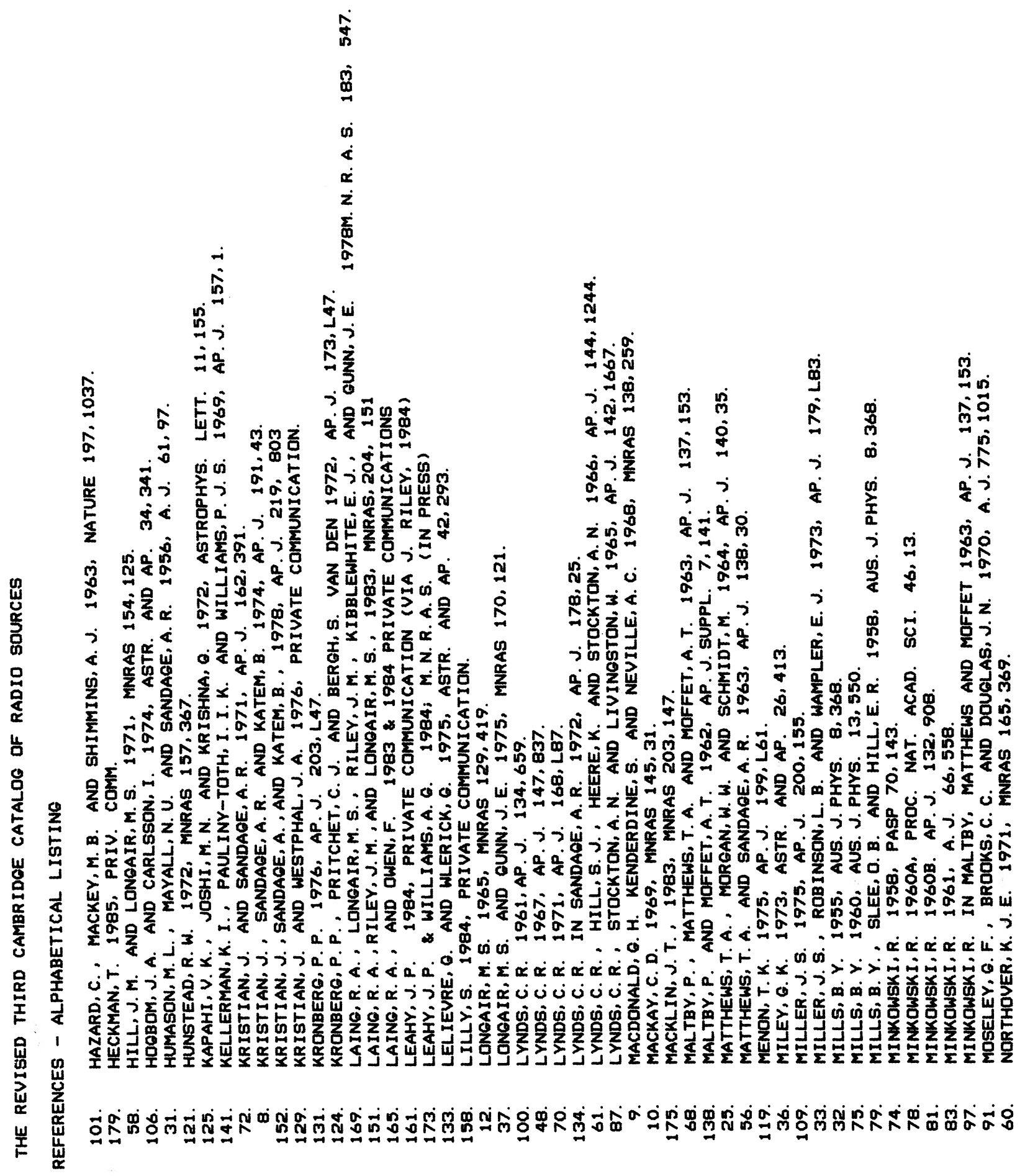




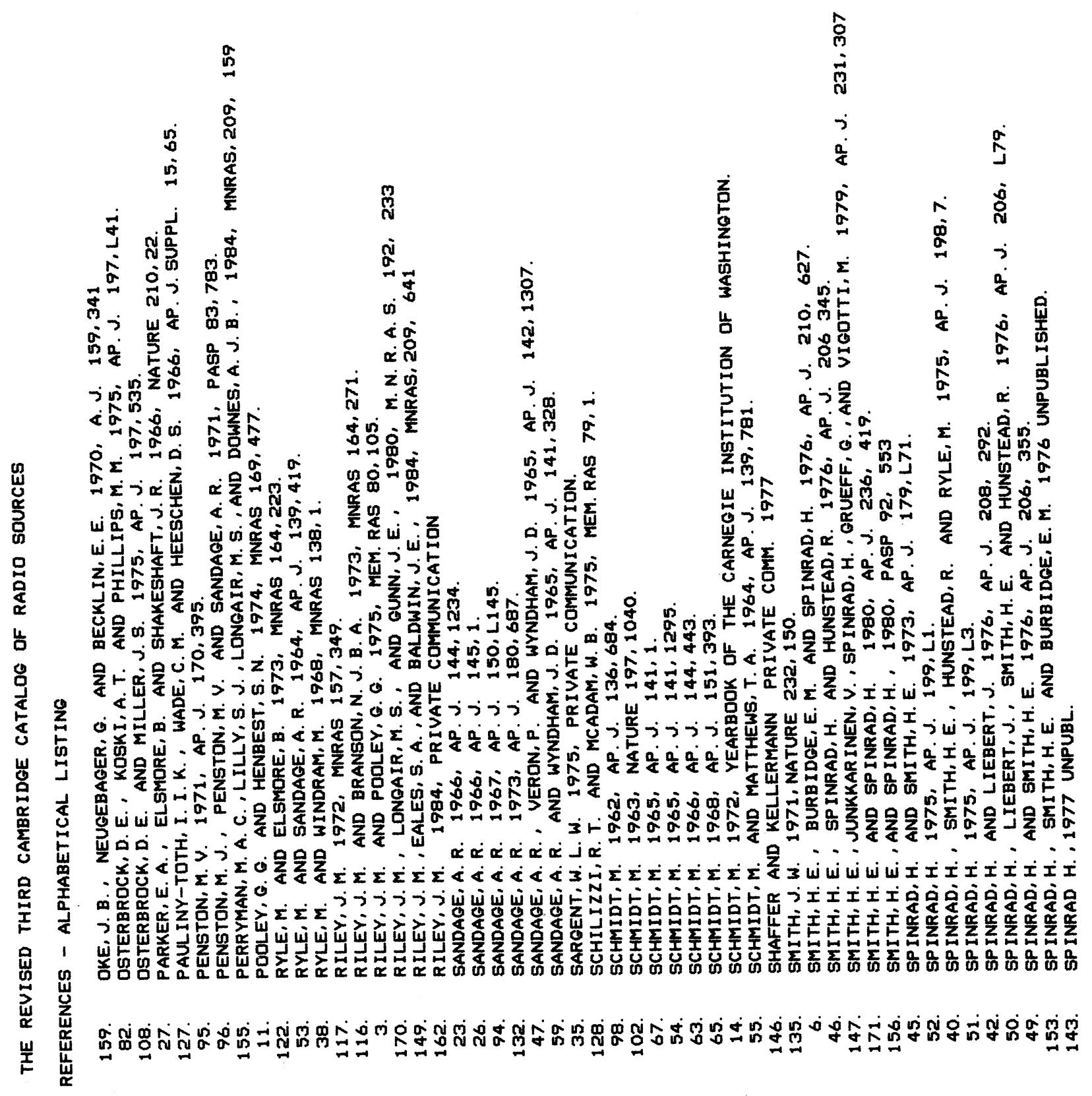


京

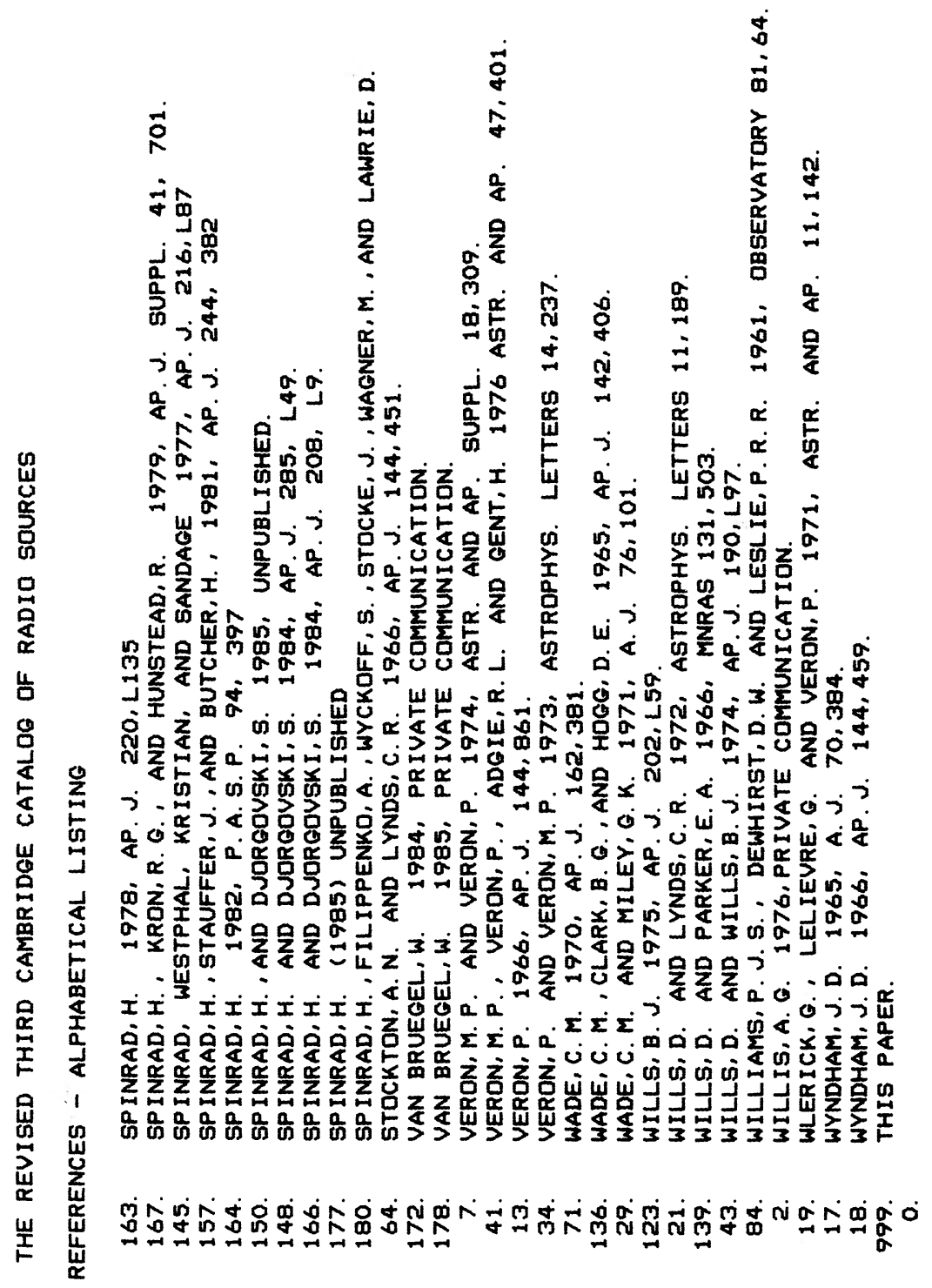




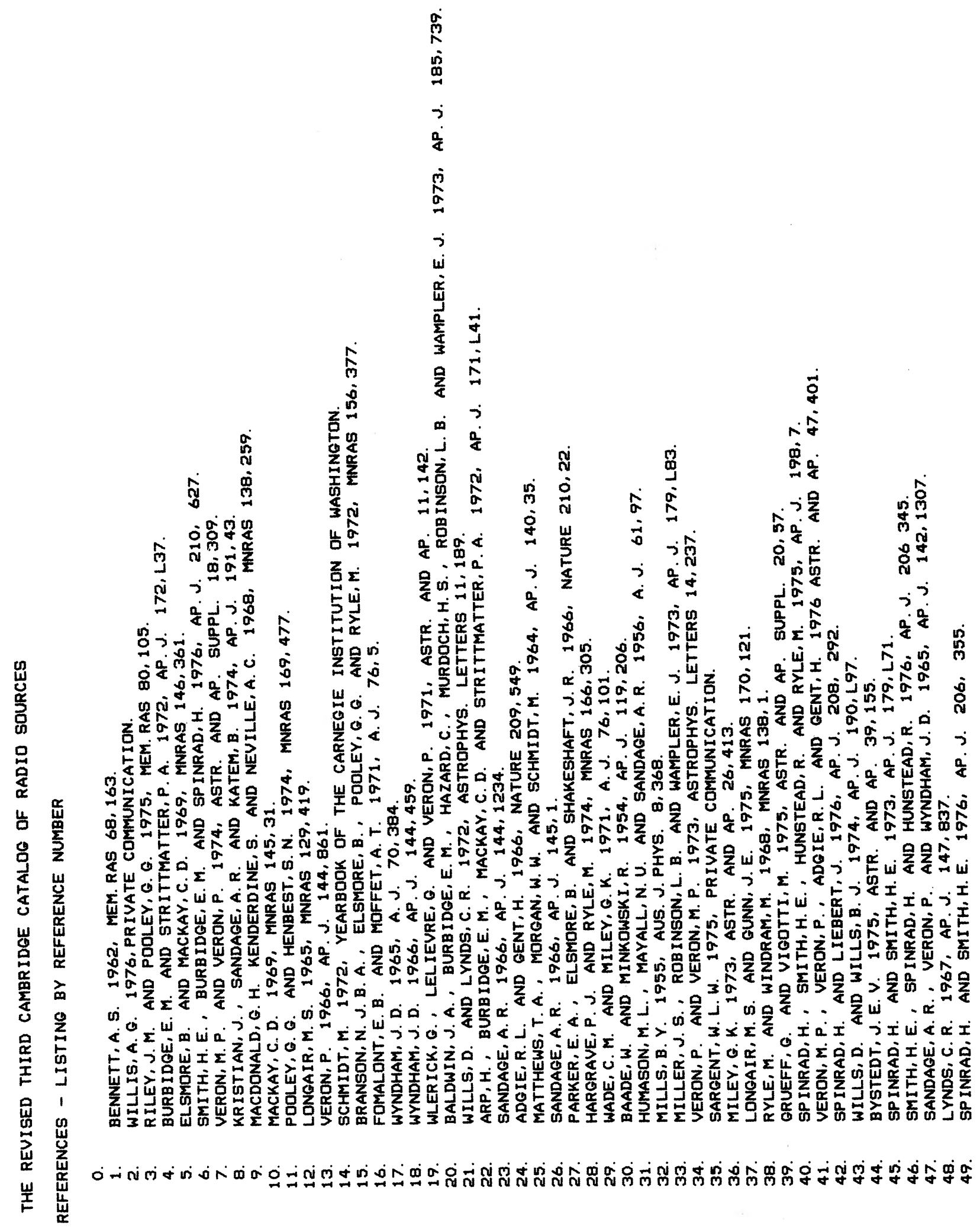




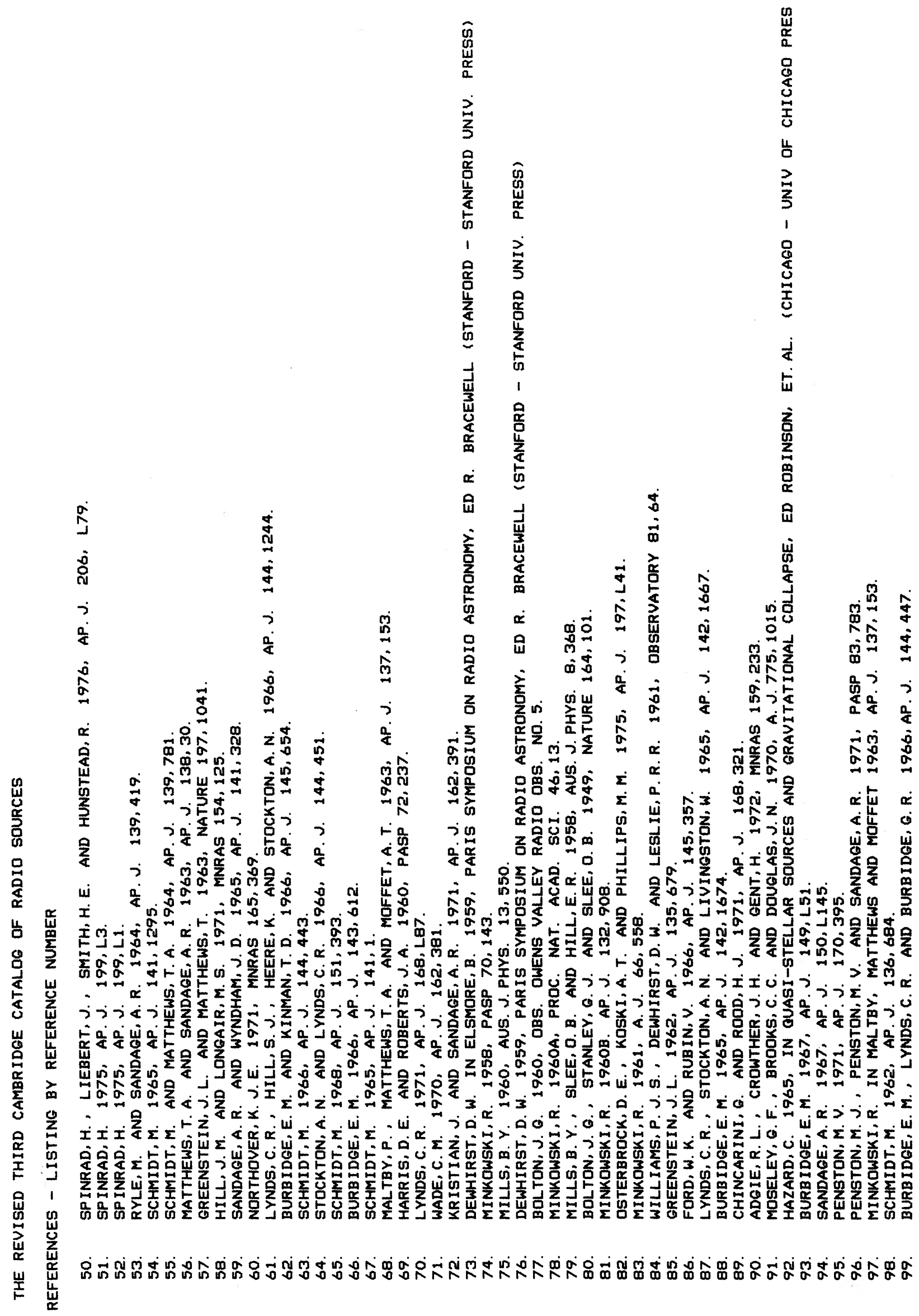




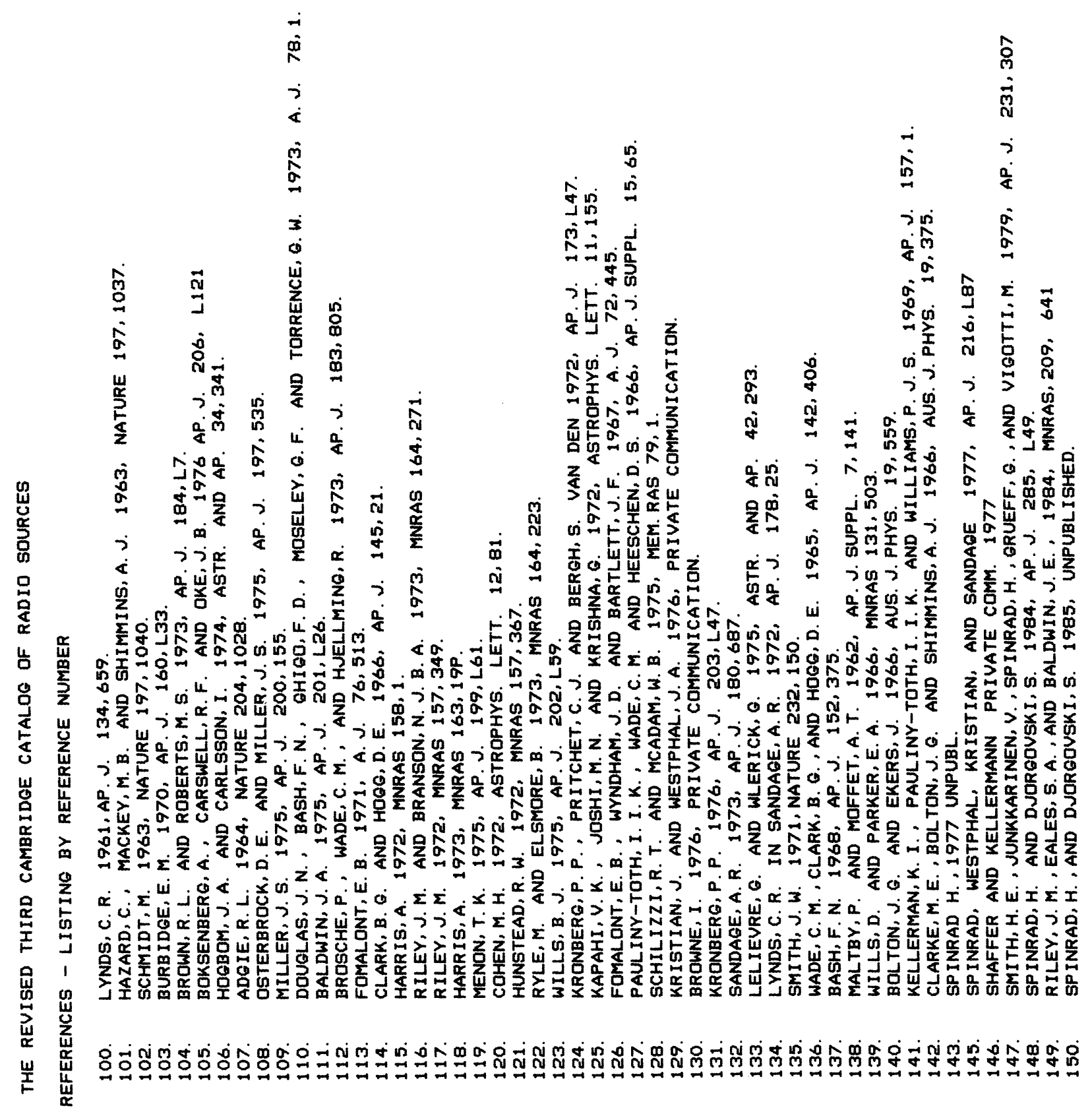




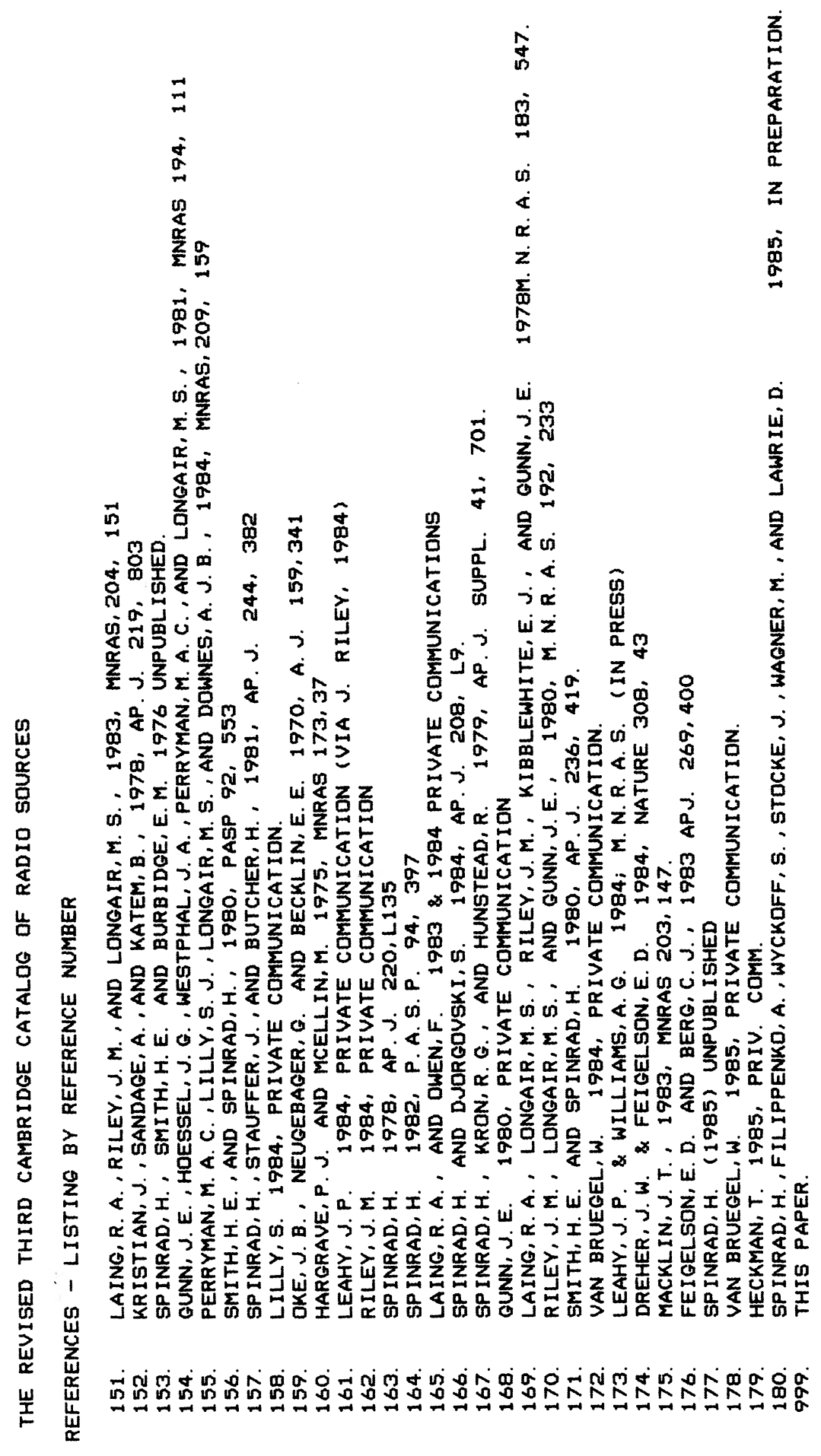

